\title{
A Finescale Radar Examination of the Tornadic Debris Signature and Weak-Echo Reflectivity Band Associated with a Large, Violent Tornado ${ }^{\circ}$
}

\author{
JANA LESAK HOUSER \\ Department of Geography, Ohio University, Athens, Ohio \\ HOWARD B. BLUESTEIN \\ School of Meteorology, University of Oklahoma, Norman, Oklahoma \\ JEFFREY C. SNYDER \\ Cooperative Institute for Mesoscale Meteorological Studies, University of Oklahoma, \\ and NOAA/OAR/National Severe Storms Laboratory, Norman, Oklahoma
}

(Manuscript received 24 November 2015, in final form 13 July 2016)

\begin{abstract}
High-resolution data of the tornadic debris signature (TDS) and weak-echo reflectivity band (WRB) associated with a large, violent tornado on 24 May 2011 in central Oklahoma are examined using a rapid-scan, $\mathrm{X}$-band, polarimetric, mobile Doppler radar. Various characteristics of these features and their evolution are examined over time intervals of $20 \mathrm{~s}$ or less. The formation of the TDS, debris fallout, and inhomogeneities in the TDS structure, are analyzed from volumetric and single-elevation observations. Constant-radius vertical cross sections of Doppler velocity, reflectivity, and copolar cross-correlation coefficient are compared at various times during the tornado's life cycle; from them it is found that the weak echo column (WEC) is considerably narrower than the TDS and the WEC is confined to the strong gradient of Doppler velocities in the tornado's core. The TDS of the mature tornado extends radially outward, bound approximately by the $40 \mathrm{~m} \mathrm{~s}^{-1}$ radial isodop.

Rapid-scan, near-surface data were collected for a period of 6 min, during which 2-s single-elevation PPI updates at $1^{\circ}$ were available at heights below $100 \mathrm{~m}$ above radar level. During this period, a WRB associated with a visually observed horizontal vortex developed east of the tornado, along the leading edge of the secondary rear-flank gust front, as the tornado was rapidly intensifying. A relationship was noted between reduced radar-observed reflectivity and increased radar-observed radial convergence/divergence in the vicinity of the horizontal vortex as it strengthened. This feature is qualitatively analyzed and hypotheses explaining its generation and structure are discussed.
\end{abstract}

\section{Introduction}

Mobile Doppler radars have been used to study the tornado since the late 1980s (e.g., Bluestein and Unruh 1989, 1993; Bluestein et al. 1995; Wurman et al. 1997). As technological upgrades are incorporated into these

Supplemental information related to this paper is available at the Journals Online website: http://dx.doi.org/10.1175/ MWR-D-15-0408.s1.

Corresponding author address: Jana Houser, Ohio University, 145 Clippinger, Athens, OH 45701.

E-mail: houserj@ohio.edu instruments, new discoveries are made, enabling a dramatically increased understanding of this phenomenon and advancing tornado science. High-resolution radars have identified a plethora of finescale structures near and within tornadoes (e.g., Fujita 1981; Bluestein and Pazmany 2000; Wurman 2002; Bluestein and Wakimoto 2003; Bluestein et al. 2004; Wurman and Alexander 2005; Bluestein et al. 2007; Wakimoto et al. 2011; Tanamachi et al. 2012; Wurman et al. 2013; Wurman and Kosiba 2013; Snyder et al. 2010, 2013). These features may have dynamical implications on tornado-scale processes (Bluestein and Pazmany 2000).

Recent dual-polarization upgrades made to many mobile and fixed-site radars have enabled a better 
understanding of microphysical processes and storm structure. Dual-polarization observations have identified several previously unobserved features including the differential reflectivity $\left(Z_{\mathrm{DR}}\right)$ column, $Z_{\mathrm{DR}}$ arc, $\rho_{\mathrm{hv}}$ rings, and tornadic debris signature (TDS), among others (e.g., Illingworth et al. 1987; Jameson et al. 1996; Ryzhkov et al. 2005; Romine et al. 2008; Kumjian and Ryzhkov 2008; Palmer et al. 2011; Snyder et al. 2013; Dawson et al. 2014; Kumjian et al. 2014; Snyder and Bluestein 2014; Snyder et al. 2015). Of the features listed above, the evolution of the TDS as manifest by the copolar cross-correlation coefficient $\left(\rho_{\mathrm{hv}}\right)$ is examined here in detail.

The TDS is a region of low $\rho_{\text {hv }}$ collocated with relatively low $Z_{\mathrm{DR}}$, a typically (though not always) moderateto high-reflectivity factor at horizontal polarization $\left(Z_{H}\right)$, and a vortex signature in Doppler velocity $\left(V_{R}\right)$, which corresponds to the location of nonmeteorological scatterers that are lofted by a tornado (Ryzhkov et al. 2005; Kumjian and Ryzhkov 2008). Since $\rho_{\text {hv }}$ tends to be particularly sensitive to the presence of nonmeteorological scatterers (such as debris) owing to the varying sizes, shapes, orientations, and dielectric constants of lofted debris targets within a radar volume, and not susceptible to instrument and transmission errors (e.g., calibration, beam blockage, and attenuation) (Ryzhkov et al. 2005; Van den Broeke and Jauernic 2014; Snyder et al. 2015), we will use $\rho_{\mathrm{hv}}$ to examine the TDS.

Rapid-scan capabilities offer another improvement in radar technology that has become more widely implemented in the last 5-10 years (Wurman and Randall 2001; Zrnić et al. 2007; Bluestein et al. 2010; Pazmany et al. 2013; Cheong et al. 2013; Isom et al. 2013). When studying atmospheric phenomena that evolve quickly [e.g., $\sim O(10) \mathrm{s}$ ] such as tornadoes, it is imperative to be able to retrieve volumetric data rapidly in order to resolve the processes associated with the evolving structures and kinematics. Recent studies have concluded that rapidly evolving features such as secondary (or internal) rearflank momentum surges (e.g., Marquis et al. 2012; Kosiba et al. 2013; Skinner et al. 2014; Schenkman et al. 2014; Kurdzo et al. 2015), descending reflectivity cores (Rasmussen et al. 2006; Kennedy et al. 2007; Byko et al. 2009), and rapidly evolving low-level vortices/ mesocyclones (Skinner et al. 2014; Houser et al. 2015; Bluestein et al. 2015) likely play a role in the processes associated with tornadogenesis, maintenance., intensification, and/or decay. Thus, the implementation of rapid-scan technology onto mobile platforms is showing promise for improving scientific understanding of tornadoes (French et al. 2013, 2014, 2015; Isom et al. 2013; Wurman et al. 2014; Houser et al. 2015) and even for aiding the development of applications relevant to operational forecasting (e.g., Tanamachi and Heinselman 2016).

While rapid-scan and polarimetric technologies are beneficial independently, their utility and potential for improving scientific understanding of tornadoes increase when combined. This study uses data from a rapid-scan X-band (3-cm wavelength) polarimetric mobile Doppler radar (RaXPol; Pazmany et al. 2013), to examine a large, violent tornado that occurred on 24 May 2011 in central Oklahoma. This case study is one of only a handful documenting a violent, long-lived tornado (Burgess et al. 2002; Alexander and Wurman 2005; Wurman and Alexander 2005; Kosiba and Wurman 2010; Palmer et al. 2011; Tanamachi et al. 2012; French et al. 2014, 2015; Snyder and Bluestein 2014; Kurdzo et al. 2015; Bluestein et al. 2015; Wakimoto et al. 2015). Additionally, this is the only study with both volumetric rapid-scan and polarimetric data acquisition for an extended period of time.

The study presented herein will provide a polarimetric, volumetric analysis of the structure of an (enhanced Fujita) EF-5 tornado over a time span of $\sim 30 \mathrm{~min}$. Visual observations suggest that tornadoes can undergo rapid changes in the size and shape of both the funnel cloud and the debris field (e.g., Houser et al. 2015; Wakimoto et al. 2016). Certain features apparent in the debris structure may be tied to dynamically and kinematically important storm-scale features such as secondary (internal) rear-flank momentum surges (Kurdzo et al. 2015). The primary questions motivating this study are the following: 1) How does the structure of the TDS change over short time increments? 2) What are the spatial and temporal relationships among the TDS, weak echo column (WEC), and Doppler velocities? 3) What are the radar-based characteristics of an observed narrow, weak-echo reflectivity band (WRB) located in the immediate vicinity of the tornado and collocated with a visually observed horizontal vortex, how did it develop and evolve, and how is it related to the tornado?

This study will examine the rapid evolution of the TDS structure. Debris fallout and other inhomogeneities/ asymmetries in the TDS, including what appears to be debris ejections (Kurdzo et al. 2015), and possibly shallow spiral inflow bands are discussed. Additionally, comparisons of Doppler velocity, $Z_{H}$, and $\rho_{\mathrm{hv}}$ are made based on vertical cross sections through the TDS/WEC. Finally, observations of the WRB are analyzed using single-elevation low-level [as low as $35 \mathrm{~m}$ above radar level (ARL)] polarimetric observations acquired every $2 \mathrm{~s}$. The study will focus primarily on rapidly changing aspects of these features from prior to tornadogenesis through the mature phase of the tornado. Information pertaining to the instrumentation and methodology 
used in the analysis is given in section 2. A brief overview of the dataset is given in section 3. Observations and analyses of the TDS and the WRB are presented in section 4 , and section 5 provides a discussion and summarizes the conclusions.

\section{Instrumentation and methodology}

RaXPol is capable of completing one $360^{\circ}$ PPI scan in $2 \mathrm{~s}$. The rapid data acquisition speed is enabled by a highspeed pedestal and by utilizing frequency hopping (Doviak and Zrnić 2006, p. 180) to obtain quasi-independent samples faster than what is possible using conventional radars, but it does not use a phased-array antenna. The $3-\mathrm{dB}$ beamwidth of the instrument is $\sim 1^{\circ}$. Although beam smearing increases the effective beamwidth to $1.4^{\circ}-1.5^{\circ}$, the data are oversampled every $1.1^{\circ}$. For the majority of the data examined in this study, the Nyquist velocity was between 30 and $33 \mathrm{~m} \mathrm{~s}^{-1}$, depending upon the specific scanning strategy employed, and the range resolution was $75 \mathrm{~m}$, oversampled every $15 \mathrm{~m}$, except for the first several minutes when range resolution was $150 \mathrm{~m}$, oversampled every $75 \mathrm{~m}$. For more details about RaXPol and the details of this dataset, the reader is referred to Pazmany et al. (2013) and Houser et al. (2015), respectively.

The majority of data used for this study are singleDoppler radar observations, which were edited to remove noise and ground clutter targets, and to dealias velocities. An objective analysis was performed to transform the edited data from a spherical coordinate system to a three-dimensional Cartesian grid and to interpolate data in the vertical between elevation angles. The gridded data were then used to analyze vertical cross sections and three-dimensional isosurfaces. A dual-Doppler analysis was also produced, despite some limitations in the dataset, to diagnose qualitatively the storm-scale horizontal wind field in the vicinity of the observed WRB just prior to its development. Details regarding objective and dual-Doppler analysis techniques and limitations are provided in the appendix.

\section{Dataset}

RaXPol deployed southwest of El Reno, Oklahoma (Fig. 1), at approximately 2015 UTC 24 May 2011, to collect data on an approaching supercell that formed just east of the dryline in western Oklahoma. By the time rapid-scan data collection began at 2036 UTC, a tornado later ranked as a 3 on the EF scale (McDonald and Mehta 2006; Edwards et al. 2013) was in progress southwest of RaXPol (Fig. 1). This tornado decayed at 2047 UTC, according to an NWS damage survey. A second tornado formed at 2051 UTC (B2 in Fig. 1) and continued through the time data collection terminated, which was at 2116 UTC. The latter tornado is the tornado of interest for the present study. Volume scans composed of $360^{\circ}$ planned-position indicators (PPIs) at nine elevation angles from $2^{\circ}$ to $18^{\circ}$ were acquired every $\sim 17 \mathrm{~s}$ from $\sim 2036$ to 2055 UTC. From 2055 to 2101 UTC, the data acquisition mode was changed to single low-elevation angle $\left(1^{\circ}\right) 360^{\circ}$ PPIs with a 2-s update increment. During this period, the tornado was within $2.5-4.8 \mathrm{~km}$ of the radar, resulting in a beam height of $\sim 20-55 \mathrm{~m}$ ARL (accounting for nonzero pitch and roll) $)^{1}$ and enabling near-ground analysis of the evolution of the TDS and the small-scale WRB associated with a horizontal vortex. During this period, the tornado rapidly strengthened and grew in width, attaining its maximum observed radarrelative Doppler velocity of $124.8 \mathrm{~m} \mathrm{~s}^{-1}$ at 2100:26 UTC. Partial beam-blockage east of the radar degraded data quality in the vicinity of the tornado as it moved northeastward through the blockage from 2100:32 UTC until data collection ceased. The magnitude of the maximum velocity was not affected by the partial beam blockage because it was outside the affected sector.

After 2101 UTC, as the tornado was moving away from RaXPol and appeared to be in its mature phase (i.e., not rapidly intensifying or weakening), the scanning strategy was changed back to volumetric rapid-scan mode. Volume scans composed of nine elevation angles from $1^{\circ}$ to $17^{\circ}$ were completed every $\sim 17 \mathrm{~s}$. Unfortunately, toward the end of the deployment, data quality east of the tornado was compromised further as a consequence of severe attenuation through the rear of the hook echo. Data collection ceased at 2116 UTC as the tornado moved beyond where the signal was attenuated to extinction. At this time, the violent tornado was still on the ground; it continued until $\sim 2135$ UTC.

\section{Observations and analyses}

\section{a. Evolution of the tornadic debris signature}

The development of a TDS associated with this tornado was atypical, complicated by debris fallout from the prior tornado. As the lofted debris (manifest by $\rho_{\mathrm{hv}}<0.8$ ) associated with the decaying first tornado was

\footnotetext{
${ }^{1}$ The radar platform was leveled using a hydraulic leveling system, but the pitch and roll of the truck were still nonzero, although only by several tenths of a degree (the exact offset varied over the deployment duration owing primarily to wind buffeting). The exact pitch and roll orientations were recorded by pitch and roll inclinometers and documented in the metadata collected. Exact beam heights were manually calculated when necessary based upon the values of pitch and roll contained within the metadata.
} 


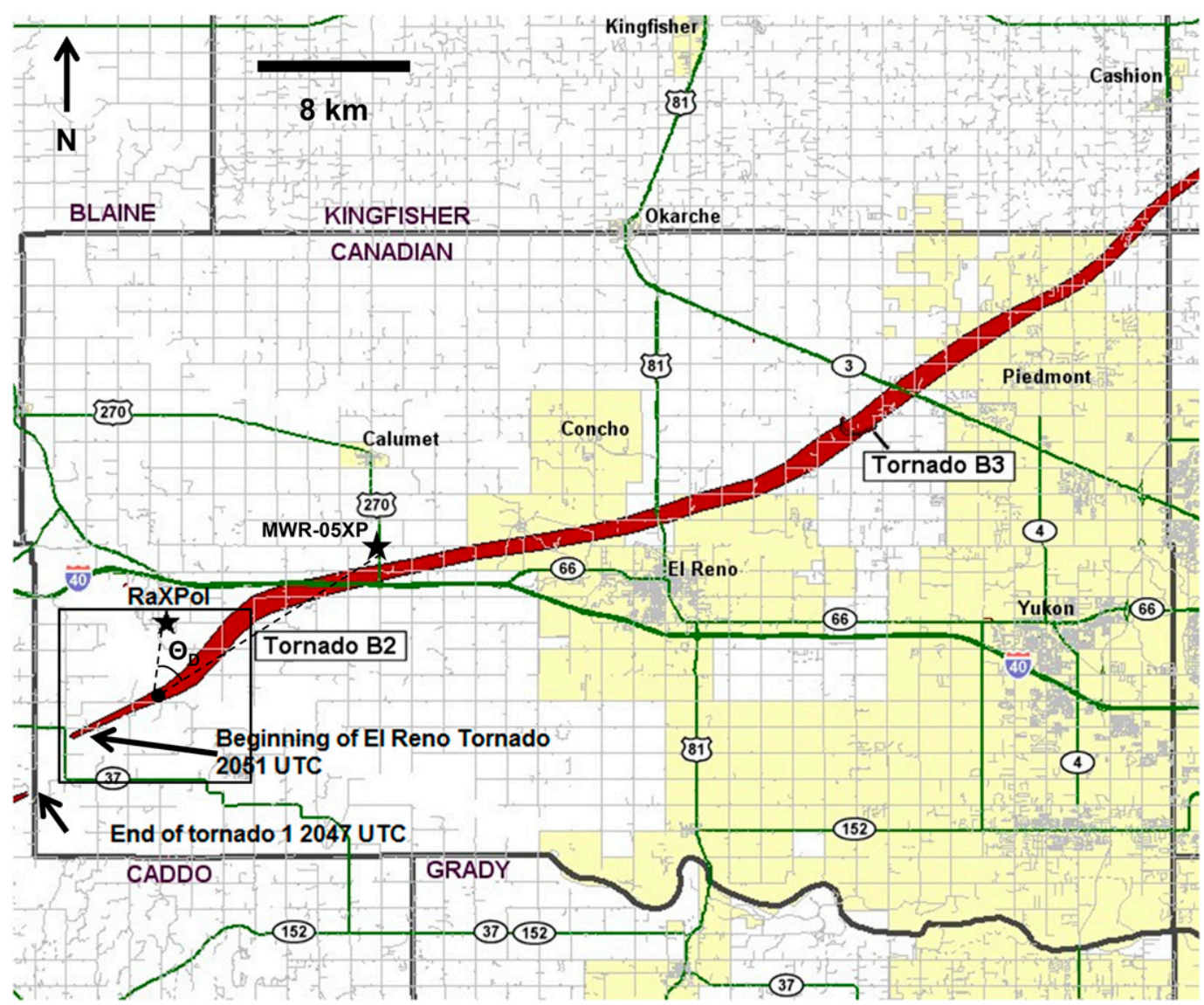

FIG. 1. Damage path of the 24 May 2011 El Reno tornado (red swath), and RaXPol and MWR-05XP deployment location (black stars). End time and location of tornado 1 are indicated as are the beginning time and location of the second tornado (B2), which is referred to as "the El Reno tornado." The square denotes the dual-Doppler grid domain for the 2056:04 UTC analysis; $\theta_{D}$ is the between beam angle of the analysis, which was $41^{\circ}$. Note tornado B3 is a satellite tornado of B2 and occurred concurrently with B2.

settling toward the ground around 2046 UTC, ${ }^{2}$ some of it was ingested back into an intensifying low-level mesocyclone to the northwest of the tornado as manifest by low values of $\rho_{\mathrm{hv}}$ progressively wrapping into the lowlevel mesocyclone, while some converged along the leading edge of the rear-flank gust front to the east and southeast of the tornado (Fig. 2a). The previously tornadic circulation fell below subtornadic ${ }^{3}$ intensity at

\footnotetext{
${ }^{2}$ The range to the tornado at this time was about $12 \mathrm{~km}$, and at the analyzed elevation angle of $4^{\circ}$, the beam height was approximately $880 \mathrm{~m}$ above radar level.

${ }^{3}$ Here and in Houser et al. (2015), the vortex was considered "subtornadic" when the maximum difference between inbound and outbound Doppler velocities $\left(\Delta V_{\max }\right)$ decreased below $50 \mathrm{~m} \mathrm{~s}^{-1}$. While this criterion is higher than others found in the literature, the observations were taken at $650-750 \mathrm{~m}$ ARL while others use lower velocity thresholds for observations taken at heights closer to the ground. The criterion employed herein was consistent with times specified in the NWS damage survey assessment.
}

2047 UTC (Houser et al. 2015), but residual debris (as manifest by regions of $\rho_{\mathrm{hv}}<0.8$ ) remained airborne and detectable by RaXPol for several minutes (Figs. 2b-d). By 2050:57 UTC the majority of the debris had fallen out (Fig. 2e), but some remaining debris was concentrated in the low-level mesocyclone. At this time, the difference between the maximum inbound and outbound $V_{r} \mathrm{~s}$ $\left(\Delta V_{\max }\right)$ from the lowest level of data that were available $\left(4^{\circ}, z \sim 650 \mathrm{~m}\right.$ ARL) exceeded the tornadic threshold of $50 \mathrm{~m} \mathrm{~s}^{-1}$ (Fig. 3a); however, a vertically coherent tornado vortex signature (i.e., a TVS present in multiple, contiguous elevation angles in the radar data) and a visual funnel cloud were not present until about $30 \mathrm{~s}$ later (Houser et al. 2015, also refer to their Fig. 5). Despite the absence of a vertically coherent vortex, a debris signature (DS) was evident in the $4^{\circ}$ elevation scan. Therefore, the tornadic-strength winds several hundred meters $A R L$ and the onset of a DS preceded the development of the vertically coherent tornado vortex signature and funnel cloud by approximately $30 \mathrm{~s}$. The observation that 

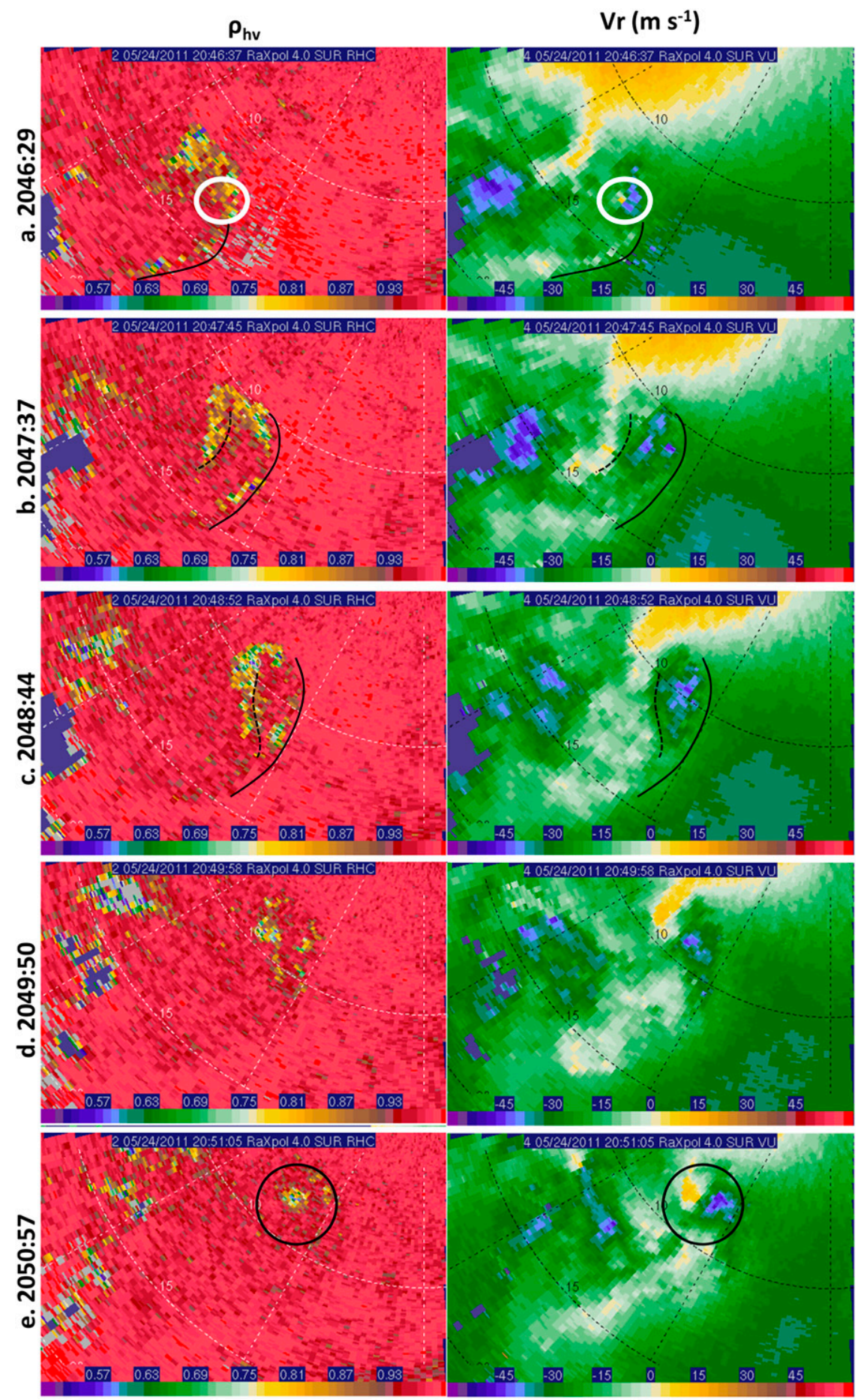

FIG. 2. (left) Copolar cross-correlation coefficient and (right) radial (Doppler) velocity every $67 \mathrm{~s}$ for the $4^{\circ}$ elevation angle (about $880 \mathrm{~m}$ above radar level) documenting the evolution of debris falling out of (a) the decaying first tornado (white circle), its accumulation along the rearflank gust front (RFGF) (black solid line) and secondary RFGF (dashed black line), and concentration in the vicinity of (e) the strengthening low-level mesocyclone (solid circle) prior to the genesis of the El Reno tornado. Range rings are plotted every $5 \mathrm{~km}$, north is up. 

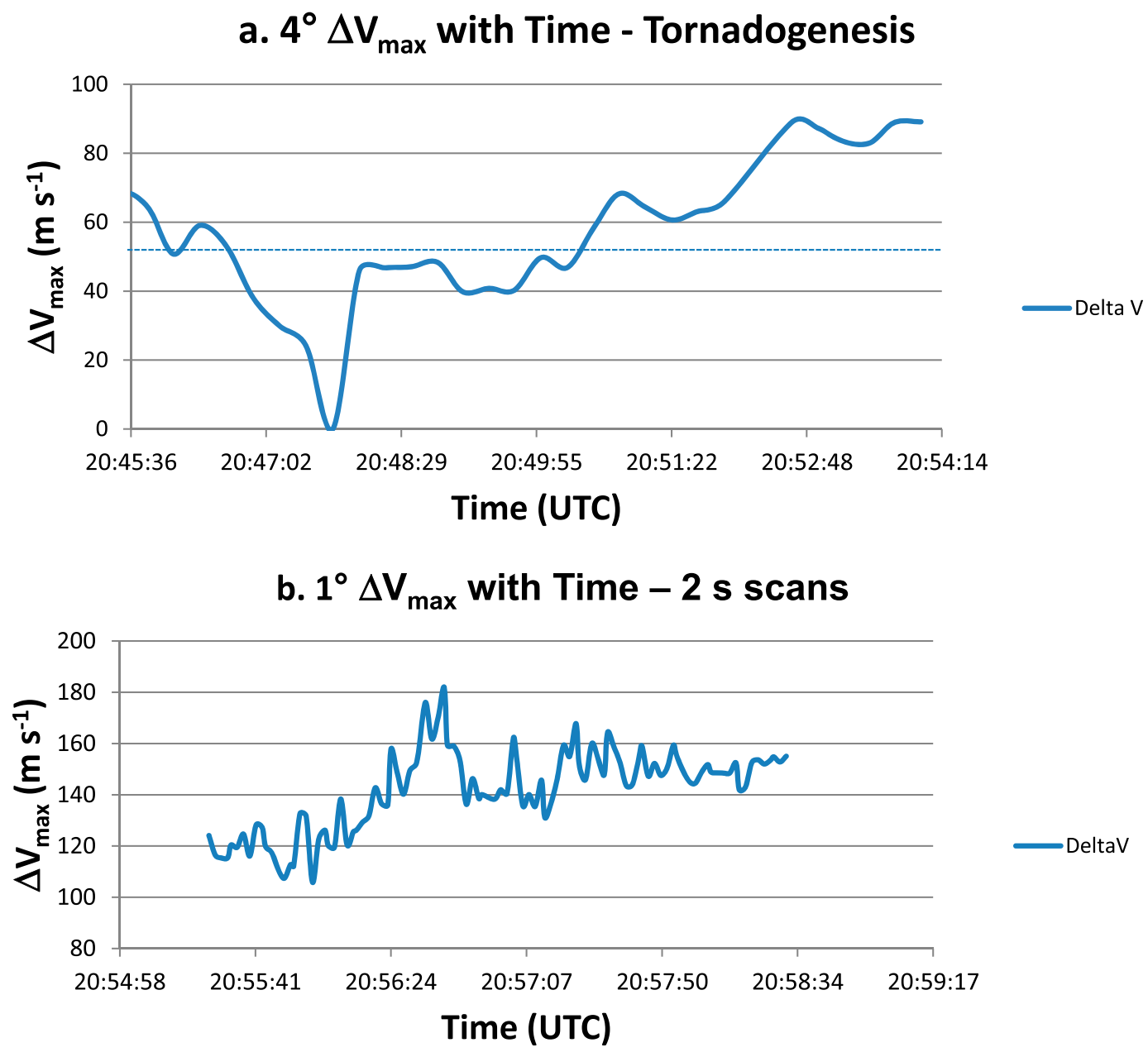

\section{c. $3^{\circ} \Delta \mathrm{V}_{\max }$ with Time}

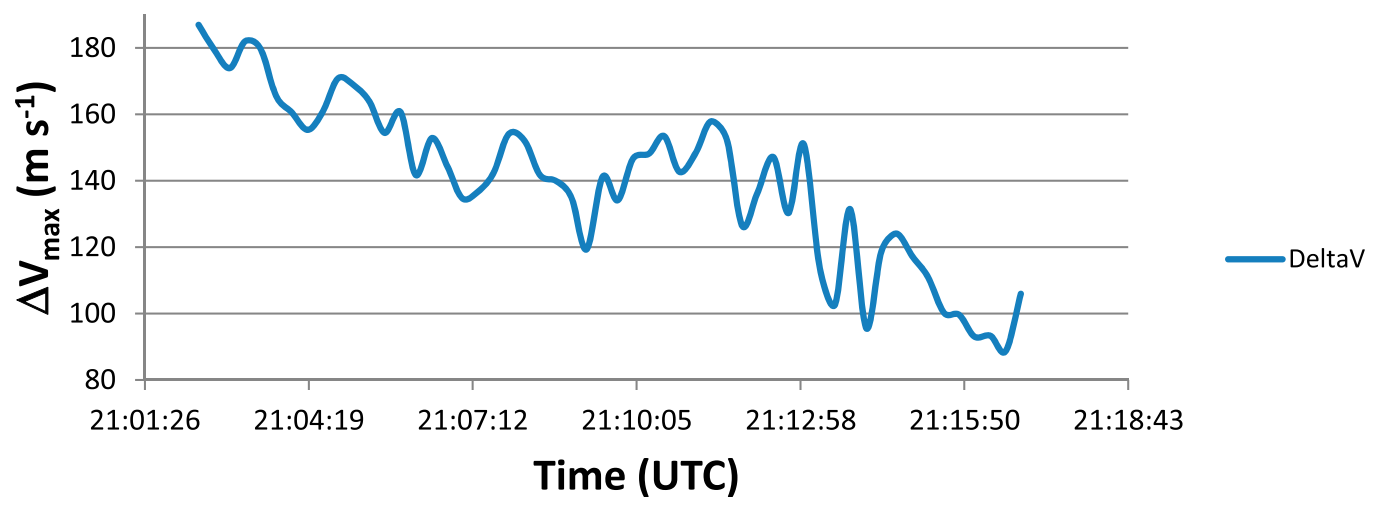

FIG. 3. Intensity trends of the tornado at low levels as manifest by $\Delta V_{\max }$ (ordinate, $\mathrm{m} \mathrm{s}^{-1}$ ) as a function of time (abscissa, UTC). Data are at different elevation angles representing the lowest elevations for which data are available given the particular scanning strategy that was employed at that time: (a) $4^{\circ}$ elevation, between $1 \mathrm{~km}$ and $400 \mathrm{~m}$ ARL, (b) $3^{\circ}$ elevation, between $\sim 100$ and $300 \mathrm{~m}$ ARL, and (c) $1^{\circ}$ elevation, between $\sim 20$ and $70 \mathrm{~m}$ ARL. Exact heights within the ranges specified are dependent upon the distance between the radar and the tornado at any given time. Dotted line in (a) indicates the $50 \mathrm{~m} \mathrm{~s}^{-1}$ threshold. 
the DS preceded the official start time of the tornado and the visual onset of the funnel cloud has also been made by others (e.g., Johnstone et al. 2012; Schultz et al. 2012; Saari et al. 2014; Van Den Broeke 2015). In the absence of velocity observations below $\sim 650 \mathrm{~m}$ at this time, it is not possible to determine whether the debris contained in the observed DS came from remnant lofted debris from the previous tornado, or from new debris lofted by the increasing low-level winds associated with the nascent tornado. However, the presence of a debris signature prior to the onset of a vertically coherent tornado implies that the presence of a fully developed tornado was not required for the development of the DS in this case.

The evolution of the debris field around the time the El Reno tornado formed is further illuminated by threedimensional isosurfaces of $\rho_{\mathrm{hv}}$ (Fig. 4). Initially (at 2049: 39 UTC), debris was scattered in various locations, with some concentrated in the vicinity of the strengthening low-level mesocyclone and some just to the southeast of this location, along the occluded rear-flank gust front (RFGF). The pattern of debris was not axisymmetric, and its vertical extent varied from about $1 \mathrm{~km}$ to just under $2 \mathrm{~km}$, implying perhaps that upward vertical motion was not spatially consistent but was strong enough to keep the debris airborne over a relatively large area, or that debris characteristics such as size/ weight were different in the different locations. Then $34 \mathrm{~s}$ later, at 2050:13 UTC, the vertical extent of the debris briefly increased to about $2.7 \mathrm{~km}$, and a debris overhang was observed extending westward from the subtornadic low-level vortex as it intensified. However, the vertical extent decreased to less than $1.5 \mathrm{~km}$ over the next minute, near the time of tornadogenesis (around 2051:22 UTC). During this minute, the distribution of debris around the circulation became more axisymmetric, presumably converging into the developing vortex and being concentrated in the region of upward motion associated with the nascent tornado. At 2052:37 UTC, just over a minute after tornadogenesis, the TDS extended to a height of almost $3 \mathrm{~km}$. As the tornado approached the radar, the upper extent of the data collection volume lowered such that the full vertical extent of the TDS could not be determined, but the vertical extent clearly increased very quickly as the tornado intensified. The TDS grew horizontally over the next two minutes and was widest at the base, generally tapering with height. At the lowest elevation angle $\left(4^{\circ}, z \sim 360 \mathrm{~m}\right.$ ARL), the TDS was about $1 \mathrm{~km}$ wide; $\rho_{\mathrm{hv}}$ was consistently less than 0.7 and the minima approached 0.4. From the three-dimensional isosurfaces, it is clear that the TDS, while roughly axisymmetric, still had threedimensional characteristics. Bulges and asymmetries were evident and change from volume to volume. After 2054 UTC, the data acquisition mode changed and volumetric data were no longer available until after 2102 UTC. From these observations, it is clear that prior to tornadogenesis, the remnant debris was spread throughout the hook echo, along and behind the primary rear-flank gust front, while during and after tornadogenesis, it was concentrated in the vicinity of the nascent tornado. Although it is beyond the scope of this paper to explain this observation in detail, it is intriguing to consider that the debris distribution may provide additional insight about the flow field in the vicinity of the tornado. In this case, presumably the strengthening convergence in the vicinity of the strengthening low-level rotation concentrated the residual debris in close proximity to the vortex.

When volume scans resumed at 2102 UTC, the tornado had become very intense. The TDS was larger in diameter, wider aloft than at the surface, and tilted to the northwest with height (Fig. 5). The difference between maximum inbound and outbound velocities $\left(\Delta V_{\max }\right)$ was over $180 \mathrm{~m} \mathrm{~s}^{-1}$ in the lowest elevation scan ( $\sim 200 \mathrm{~m}$ ARL) (Fig. 3c). The TDS was approximately $1.5 \mathrm{~km}$ in diameter at this height, and $\rho_{\mathrm{hv}}$ was consistently $<0.5$ with a minimum approaching 0.1 . For three volume scans, a short-lived maximum in $\rho_{\mathrm{hv}}$ was observed in the central part of the TDS at heights $>1.25 \mathrm{~km}$ (Fig. 6). This feature is similar to what was observed by Bodine et al. (2014) and Wakimoto et al. (2015). Although not enough information is available to know for certain what is responsible for this increase in $\rho_{\mathrm{hv}}$, it is hypothesized that the presence of raindrops or a relative lack of debris near the central axis of the tornado results in a local maximum in $\rho_{\mathrm{hv}}$. Wavelike asymmetries in $\rho_{\mathrm{hv}}{ }^{4}$ (also visible in $V_{r}$ ) perhaps a manifestation of centrifugal waves (Shapiro 2001), are seen propagating up the periphery of the TDS in Fig. 5. One of the waves was tracked with time, ascending approximately $2.2 \mathrm{~km}$ over $65 \mathrm{~s}$, implying a vertical propagation speed of $\sim 33 \mathrm{~m} \mathrm{~s}^{-1}$. Wavelike features have also been observed visually along the edge of funnel clouds (Pauley and Snow 1988; Bluestein et al. 2015) and in laboratory-generated vortices (Church et al. 1979), but it is unclear whether or not the waves mentioned in the previous studies and the perturbations in $\rho_{\mathrm{hv}}$ observed herein are a result of the same

\footnotetext{
${ }^{4}$ These features generally evolve smoothly with time and do not seem to be associated with artifacts caused by finite radar beamwidth or with the gridding/radius of influence criteria for the objective analysis. Also, bulges in the raw radar data are evident at the same time these features are observed in the objectively analyzed data. Thus, we do not believe these features are spurious.
} 
2049:39

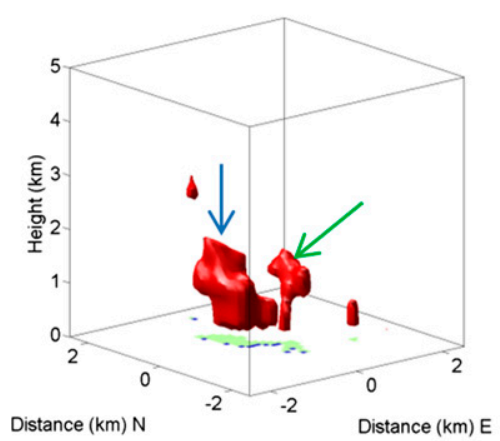

Tornadogenesis - 2051:20

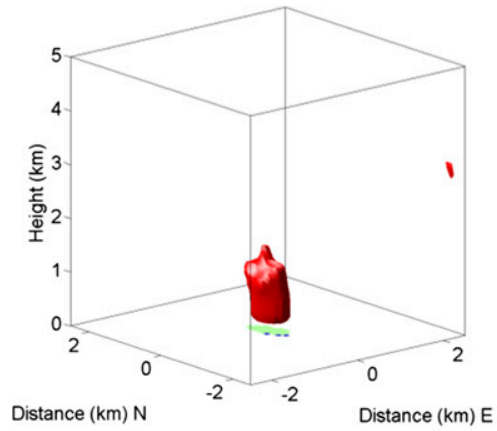

2053:09

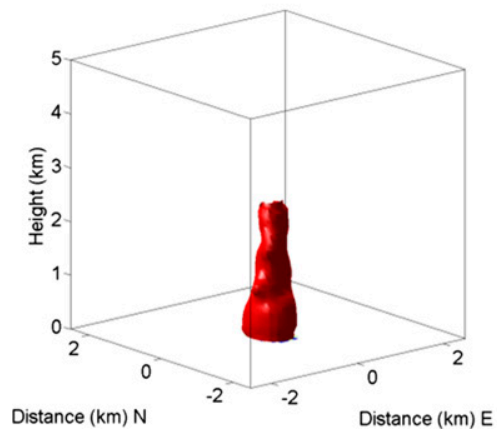

2050:13

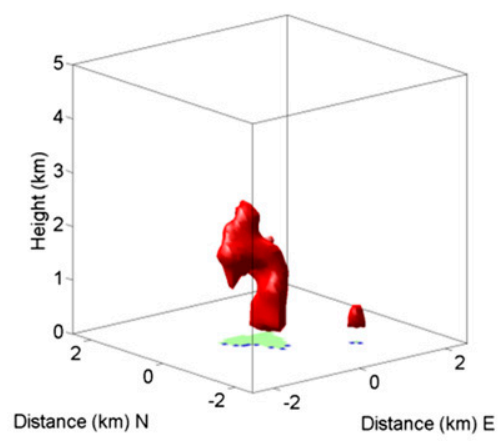

2051:53

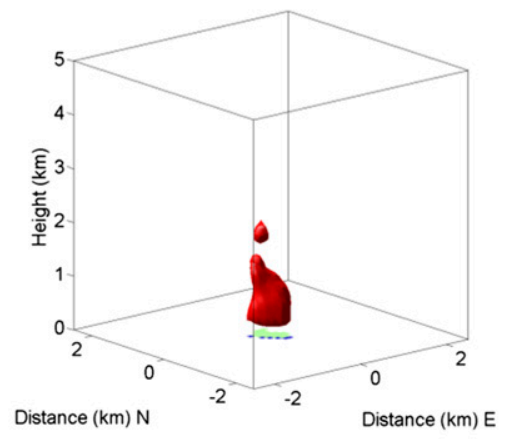

2053:42

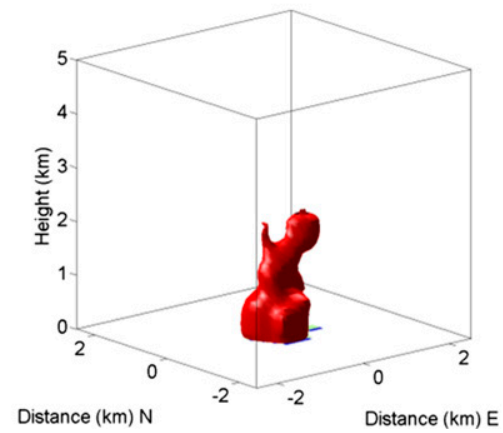

2050:46

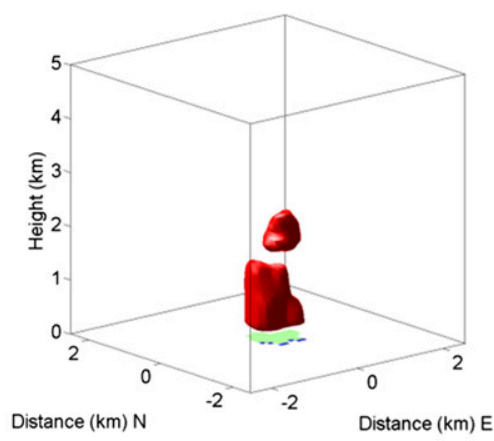

2052:37

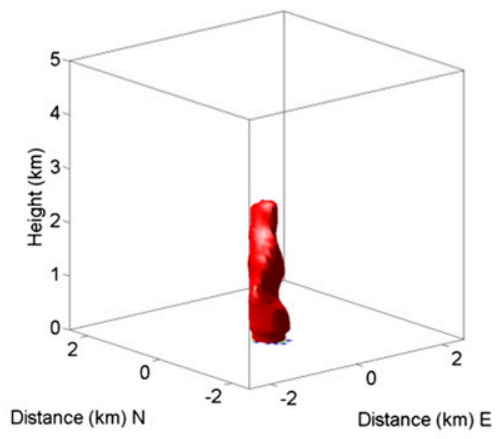

2054:15

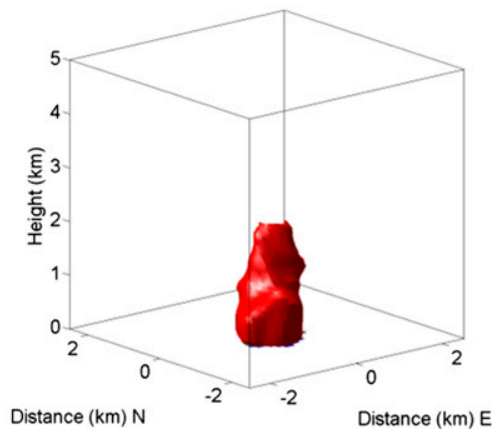

FIG. 4. Three-dimensional (west-east axis on the right, east is directed to the right; north-south axis on the left, north is directed to the left; vertical distance on $y$ axis) isosurfaces of $\rho_{\text {hv }}$ (the 0.8 isosurface is plotted) illustrating the evolution and structure of debris in the vicinity of the nascent El Reno tornado prior to genesis and afterward. The time increment between all panels is the time it takes to complete two volume scans, or approximately $33 \mathrm{~s}$, except between 2050:53 and 2052:37 UTC when the scanning strategy was changed, which interrupted the data acquisition rate. The light green and blue pixels periodically visible on the bottom of the panels represent a top-down projection of the TDS shadow onto the surface plane. The viewing angle is from the southwest toward the northeast. The blue arrow at 2049:39 UTC denotes the location of the low-level mesocyclone and the green arrow denotes the location of the RFGF.

physical processes or if they have any dynamical implications for the tornado's behavior.

As the tornado moved away from the radar and the upper limit of the data collection volume near the tornado increased, the vertical extent of the TDS became more apparent. At 2111 UTC, the TDS still extended through the top of the domain, above $5 \mathrm{~km}$, and showed no evidence of decaying with height (Fig. 7).
Between 2111 and 2116 UTC, the strength of the lowlevel ( $3^{\circ}$ elevation angle) TVS, as measured by $\Delta V_{\max }$, decreased from 157 to $88 \mathrm{~m} \mathrm{~s}^{-1}$ (Fig. 3c). This weakening trend was also observed at all other heights (not shown), suggesting the tornado itself weakened over this period. Coincident with this trend in $\Delta V_{\max }$, debris fallout from the TDS was evident (Fig. 7). Prior to the onset of this weakening trend, the TDS was quite wide 
2102:37

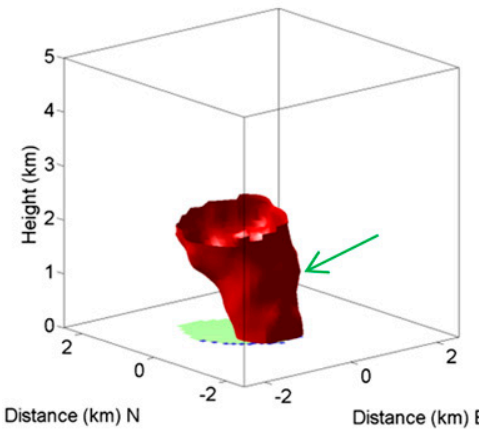

2103:26

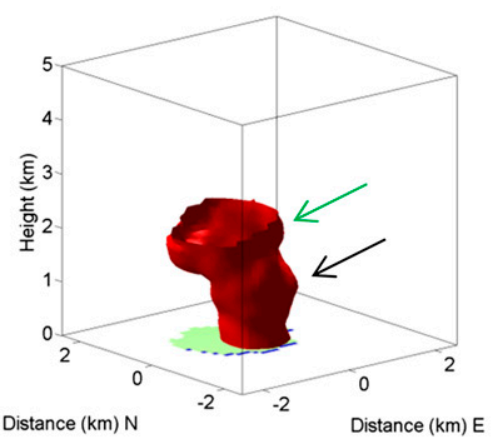

2102:54

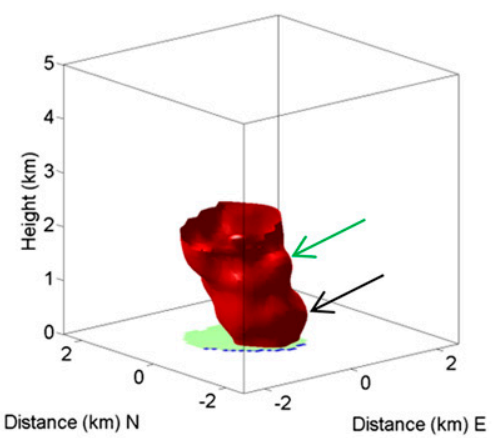

2103:43

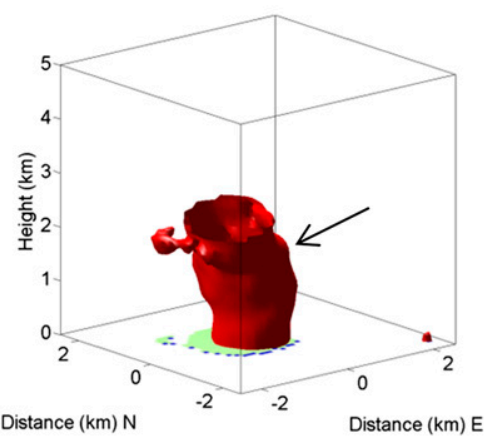

2103:10

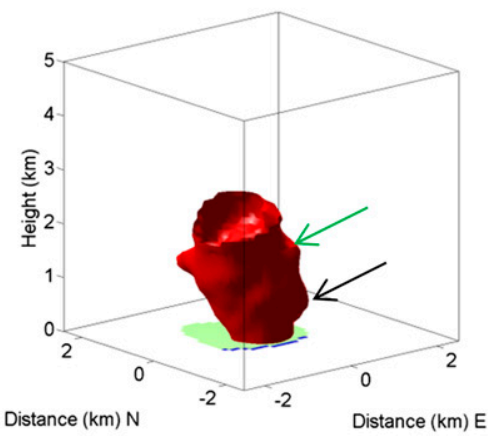

2103:59

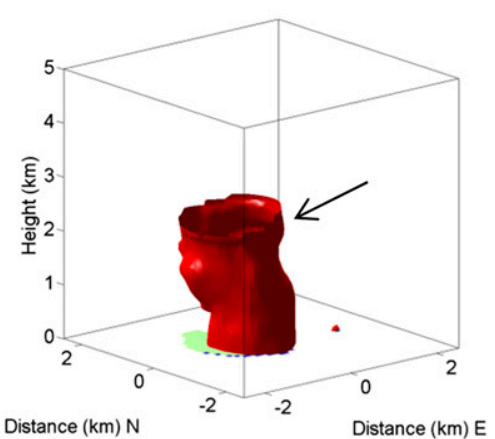

FIG. 5. As in Fig. 4, but between 2101:37 and 2103:59 UTC, and for every volume scan (time step is $\sim 17$ s). The evolution of wavelike bulges up the edge of the TDS is also shown. Individual wave crests are tracked with time by the green and black arrows.

at all heights, perhaps being slightly wider at the top than at the bottom (2109:43 and 2110:32 UTC). However, over the next several minutes, the upper portion of the TDS became progressively narrower while the middle and lower portion became wider. By 2116:32 UTC, the vertical extent of the TDS had decreased to approximately $2 \mathrm{~km}$, and its diameter had grown to nearly $4 \mathrm{~km}$. These observations support the notion of lofted debris that had been suspended, fell out, and was centrifuged or diverged outward around the lower portion of the tornado. Because the decrease in $\Delta V_{\max }$ occurred just prior to when the debris signature widened at low altitudes and disappeared above, it is hypothesized that as the tornado weakened, its central updraft also weakened, causing the lofted debris to settle out of the tornado. Reduced surface convergence associated with the weakening vortex may have also contributed to the widening of the TDS. Additionally, lateral displacement from the storm-scale updraft could contribute to these observations.

Some studies have loosely linked the size and/or height of a TDS with the tornado's intensity (Van Den Broeke and Jauernic 2014; Kurdzo et al. 2015; Van Den Broeke 2015). In this case, the simultaneous decrease in vertical extent and increase in horizontal diameter while the tornado weakened are evidence that the width of the TDS is not necessarily indicative of the strength of the tornado, as the tornado in this case was stronger when the TDS diameter was narrower.

\section{b. Asymmetries in the TDS}

Shortly after the single-elevation scans began (around 2056 UTC), the low-level TDS became asymmetric and was no longer a quasi-circular local minima in $\rho_{\mathrm{hv}}$. Sawtooth-like appendages became apparent and developed into small $[\sim O(100) \mathrm{m}]$ semispiral bands of low $\rho_{\text {hv }}$ located on opposite sides of the main TDS (Fig. 8). In general, there were usually only two appendages, and they were located approximately $180^{\circ}$ from each other, but there were times when four or more spiral bands were present. From animations of the 2-s update data (see the online supplemental material, WRB animation), it is evident that the sawtooth features rotated cyclonically around the periphery of the TDS and were trackable individually for about $10-45$ s. Upon their decay, the sawtooth features did not break off from the main TDS or eject outward; rather, they merely disappeared. Thus, they may have been associated with shallow, narrow bands of asymmetric, accelerated inflow picking up dust and debris. It was thought that the 


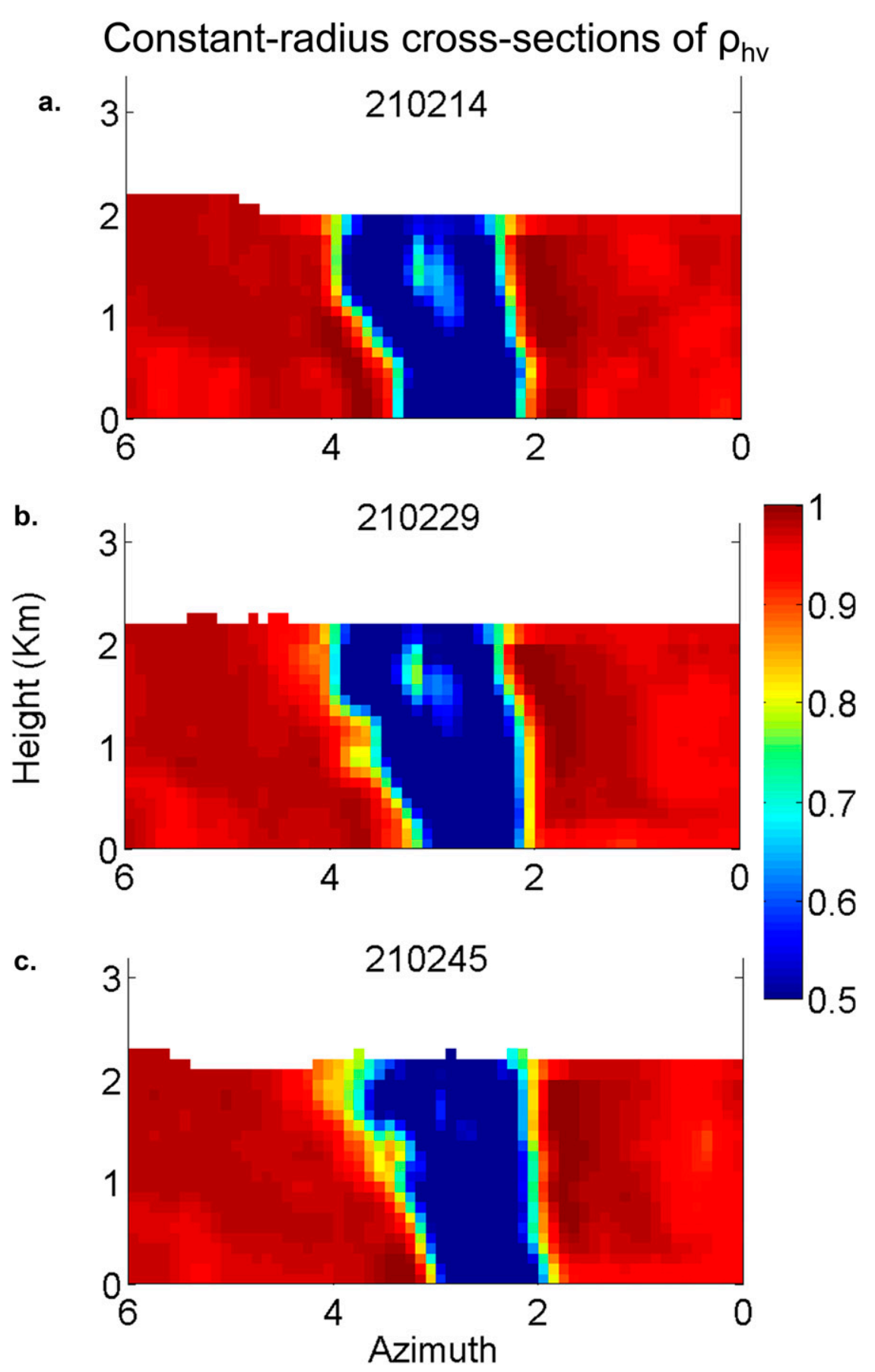

FIG. 6. Height (ordinate, $\mathrm{km}$ ) vs azimuthal arc-length (abscissa, km) cross sections of objectively analyzed $\rho_{\mathrm{hv}}$ through the tornado at constant range: (a) 4.9 , (b) 5 , and (c) $5.2 \mathrm{~km}$. The zero value on the abscissa represents the azimuthal location of the radar truck at range $=0$. Times of analyses are given in UTC (hhmmss).

roughly $180^{\circ}$ orientation could have been caused by the tornado passing over a linear band of debris, like a dirt road. However, using Google Earth, it was determined that no obvious features like roads appeared to be collocated with the observance of the sawtooth features.
Although visual observations were not available because the tornado was enshrouded by precipitation at this point, the polarimetric structure bears striking resemblance to bands of inflow dust documented in Bluestein et al. (2016) (Fig. 8e). 
2109:43

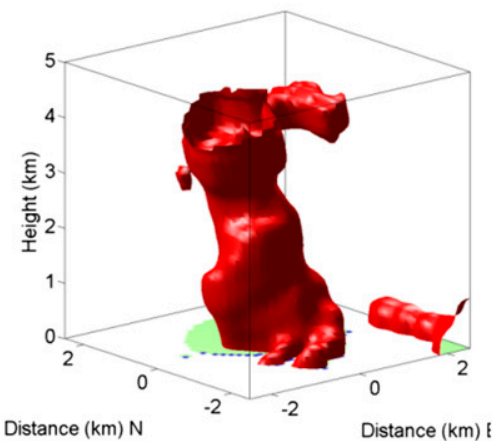

2112:27

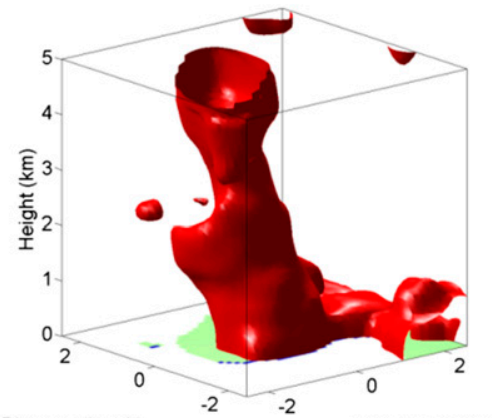

Distance $(\mathrm{km}) \mathrm{N}$

\section{4:54}

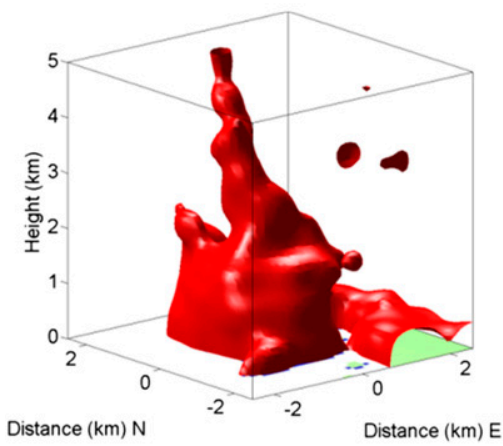

2110:32

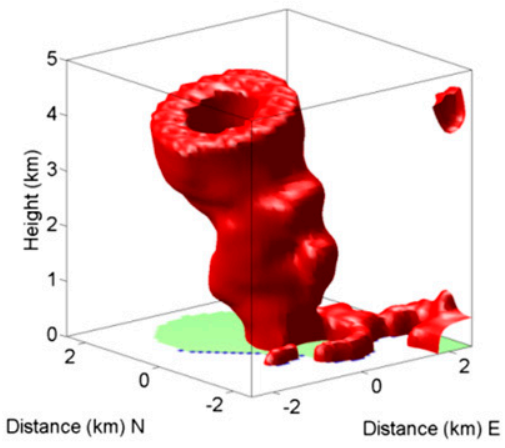

2113:16

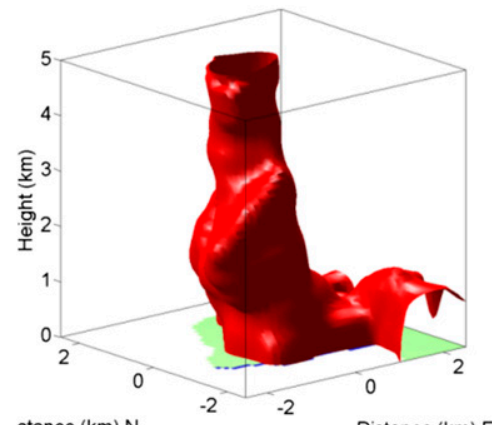

stance $(\mathrm{km}) \mathrm{N}$

2115:43

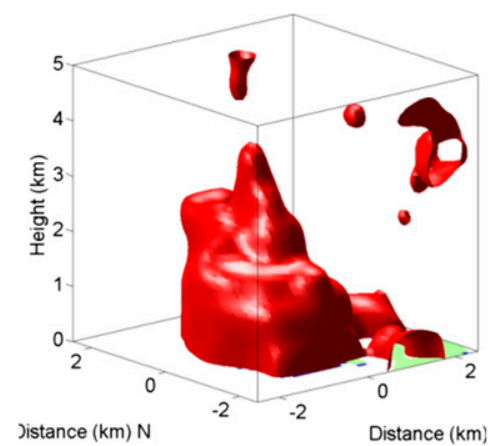

2111:38
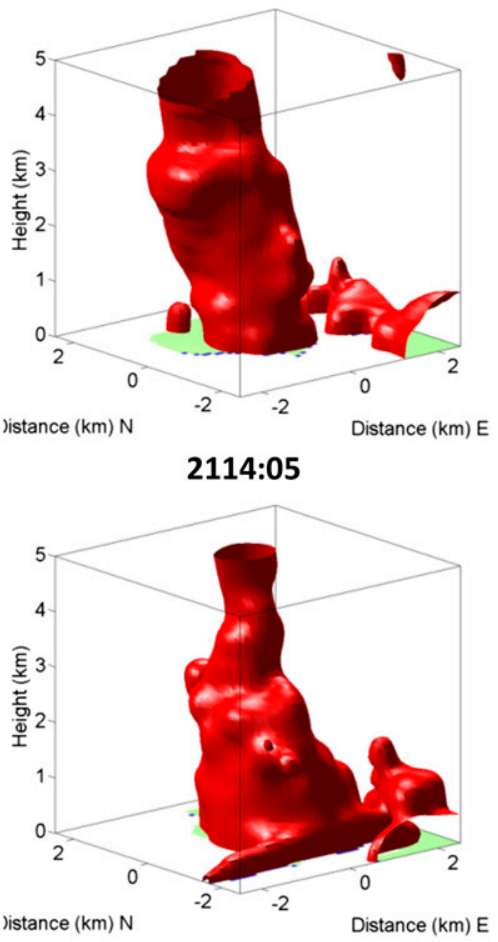

2116:32

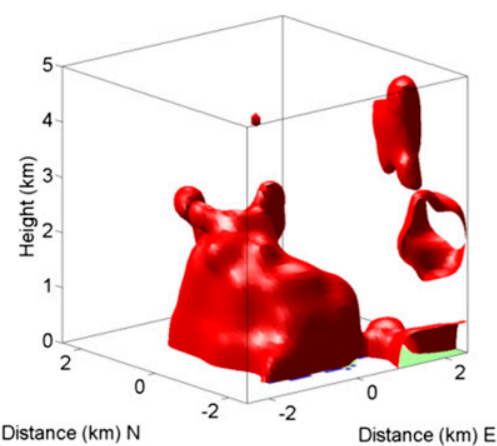

FIG. 7. As in Fig. 4, but for the evolution of the TDS from 2109:43 to 2116:32 UTC, just prior to the termination of data acquisition. Debris fallout is evident from 2113 to 2116 UTC, as the TDS becomes wide at low levels and vertically shallower. The time increment between adjacent panels is $\sim 49$ s, or every three volume scans.

At 2058 UTC, there was a signature in the $\rho_{\mathrm{hv}}$ field that resembled the "debris ejection" signature documented by Kurdzo et al. $(2015)^{5}$ in the violent tornado that destroyed parts of Moore, Oklahoma, on 20 May 2013. Herein, this feature was observed as a well-defined comma-shaped appendage to the TDS spiraling outward from the southeastern quadrant of the TDS and bending

\footnotetext{
${ }^{5}$ For consistency with Kurdzo et al. (2015), we will also call the feature observed here a "debris ejection." However, no trajectory analyses were done to determine the kinematic or dynamic processes occurring during the appearance of this feature.
}

southwestward (Fig. 9a). This signature wrapped cyclonically around the center of the TDS, and its "tail" elongated to the south with time. The tail began to separate from the main TDS at 2057:47 UTC and had separated completely by 2058:58 UTC (not shown). A subsequent but smaller debris ejection began at 2058:38 UTC, and three additional ejections occurred over the next 3 min (not shown). Kurdzo et al. (2015) correlated these events with RFGF surges (both primary and secondary) (e.g., Wurman et al. 2007; Marquis et al. 2008; Lee et al. 2012; Skinner et al. 2014). In this case, there was not an obvious zone of convergence within the radial velocity field for any of the debris ejections (e.g., Fig. 9a). 

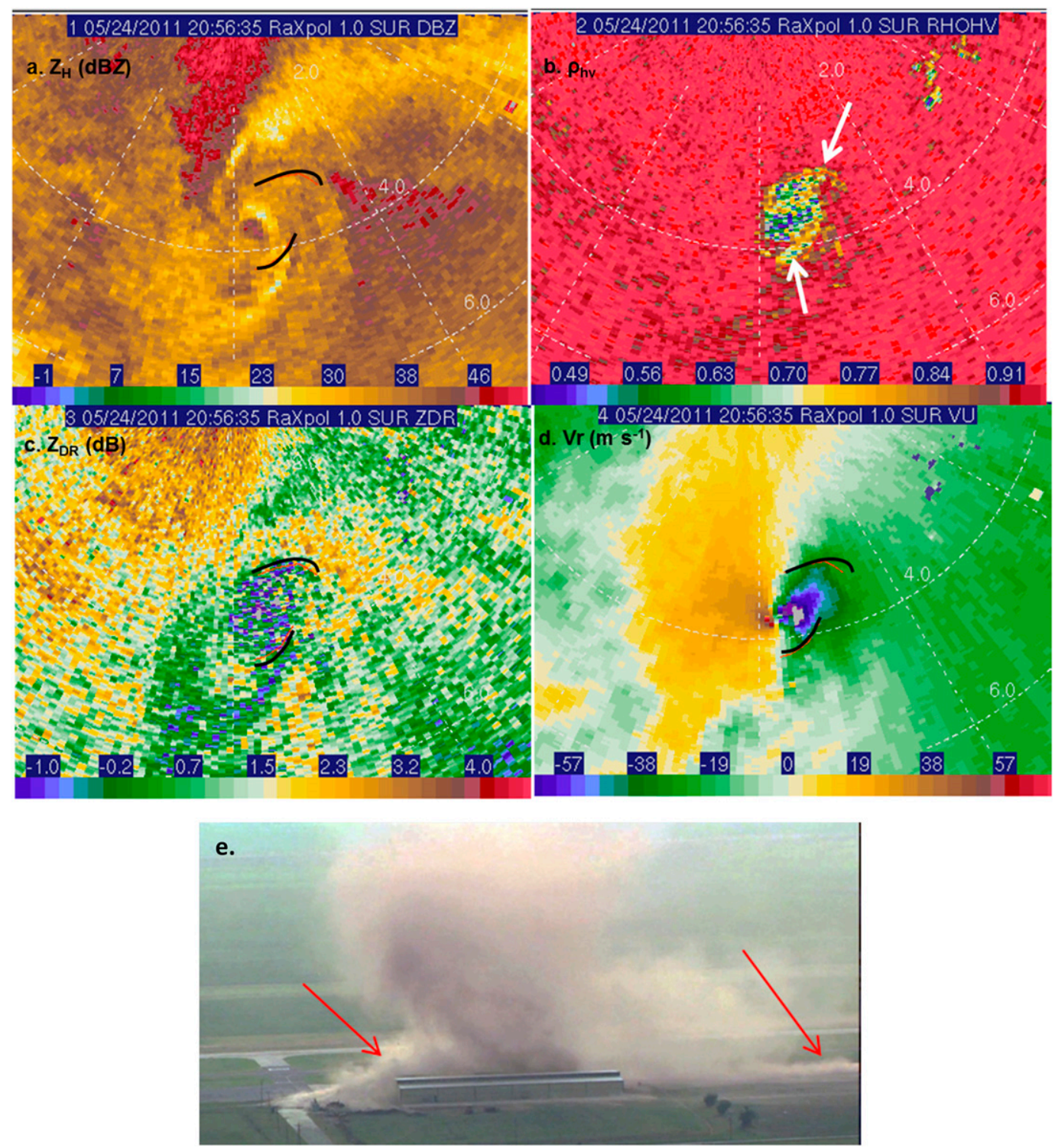

FIG. 8. (a)-(d) Example of two sawtooth features (white arrows) in the TDS and their relationship to other radar parameters (outlined by solid black lines), from the $1^{\circ}$ elevation angle scan at 2056:35 UTC. Range rings are every $1 \mathrm{~km}$. (e) Visual observation of spiral inflow bands (indicated by red arrows) taken from a still video captured by a KWTV news helicopter from a tornado that occurred on 24 Apr 2006, also near El Reno, OK; from Bluestein et al. (2016, their Fig. 2b).

It is possible, however, that the locations of the debris ejections relative to the radar were such that the velocity gradient associated with a RFGF surge was perpendicular to the radar beam and thus was not detectable in radial velocity. Regardless, the rapid sequence of these debris surges is remarkable and raises questions regarding the potential dynamical implications they may have on (or what they may indicate about) tornado and mesocyclone intensity and longevity.

The next major debris ejection occurred at 2106 UTC and was clearly associated with a secondary rear-flank momentum surge (Fig. 9b). It was farther north and east (relative to the TDS) than the previous ones, wrapping from the north side of the TDS to the southeast. From the three-dimensional isosurfaces of $\rho_{\mathrm{hv}}$ it is seen that this debris ejection had three-dimensional structure, sloping up to a height of about $1.2 \mathrm{~km}$ as the low- $\rho_{\text {hv }}$ band wrapped into the main TDS (Fig. 10). The debris quickly settled out along the trailing edge of the ejection tail but remained attached to the TDS at its head. During this process, the $\Delta V_{\max }$ at low levels $\left(3^{\circ}\right)$ fluctuated modestly (by approximately $\pm 10 \mathrm{~m} \mathrm{~s}^{-1}$ ), but 


\section{a. 2058:40}

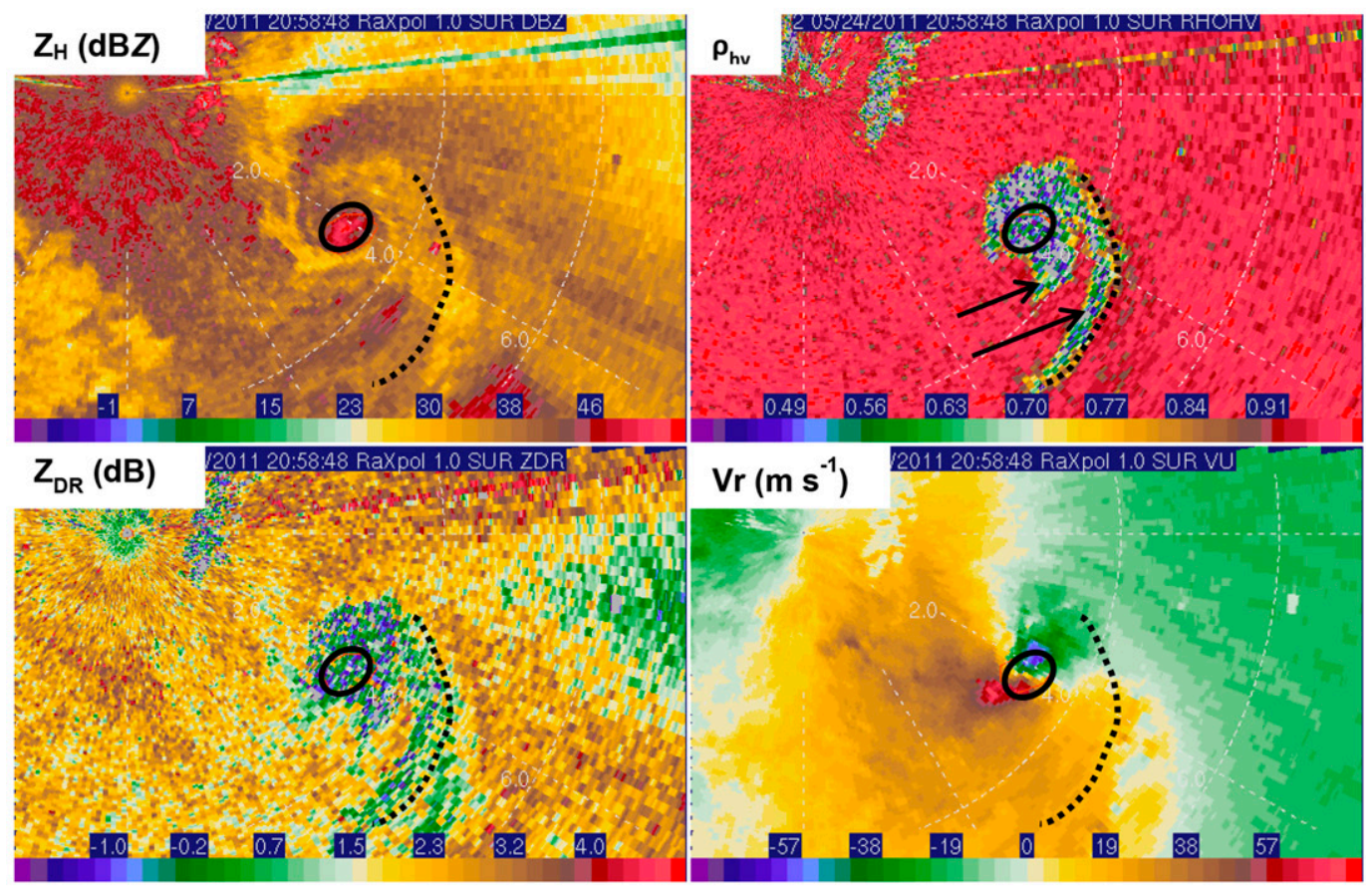

\section{b. $2107: 10$}
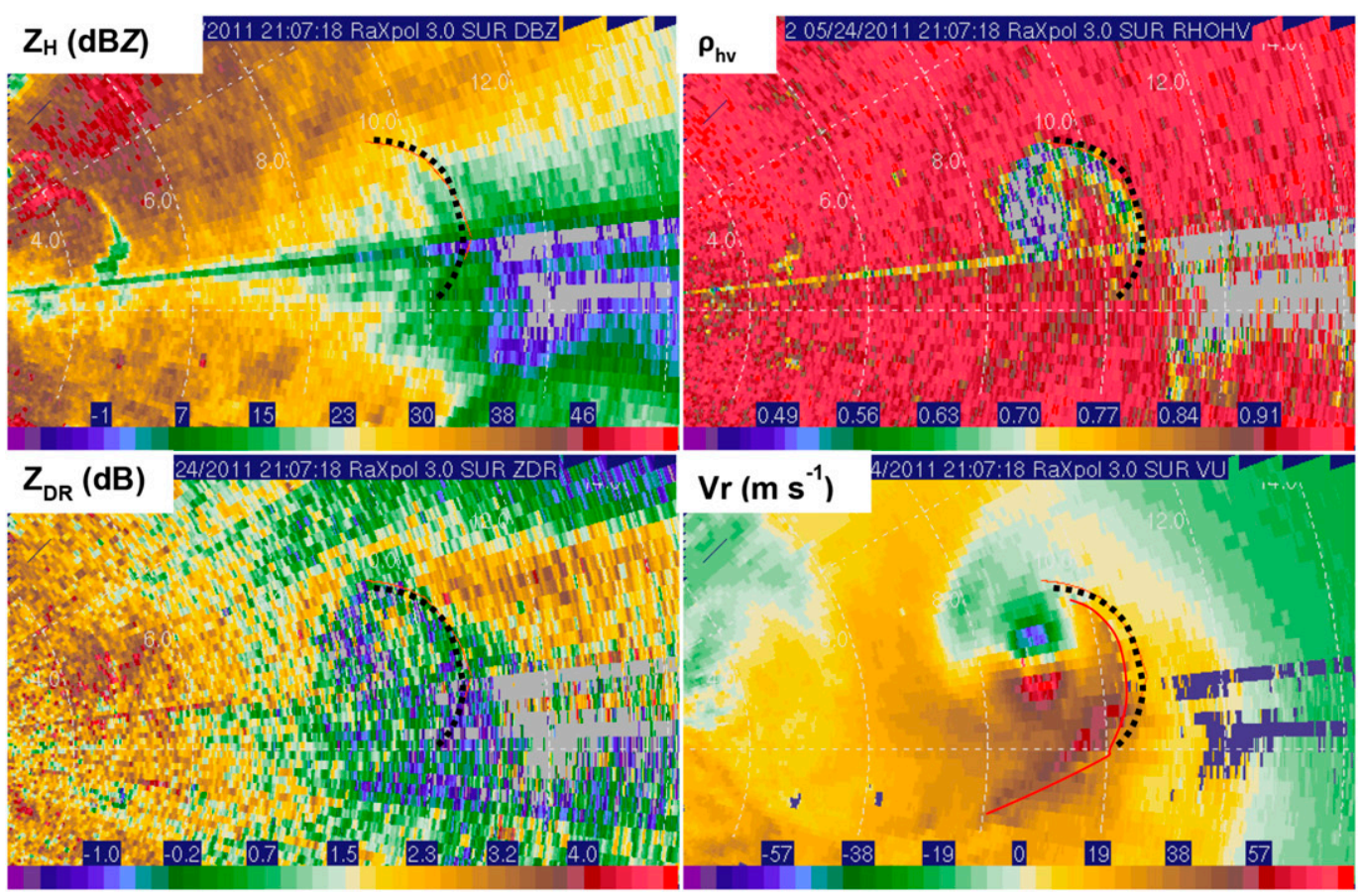

FIG. 9. Debris ejections at (a) 2058 and (b) 2107 UTC. The leading edge of the reduced $\rho_{\text {hv }}$ is indicated by the dotted curve. Relationship with the debris ball (solid ellipse) is also shown in (a). Debris ejections are indicated by the arrows in the top-right panel of (a). Beam height is $\sim 50 \mathrm{~m}$ ARL at the tornado in (a) and about $170 \mathrm{~m}$ in (b). The location of a secondary surge in momentum behind the RFD is indicated by the thin, solid red line. Both $Z_{H}$ and $Z_{\mathrm{DR}}$ are compromised in (b) as a result of partial beam blockage and significant attenuation. 


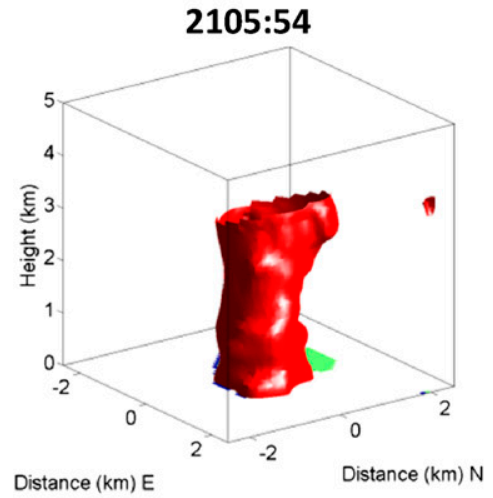

2106:43

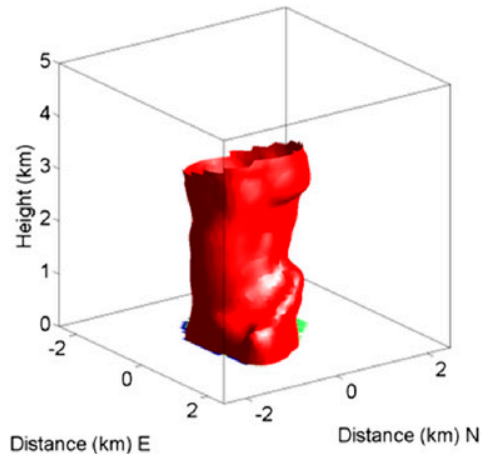

2107:32

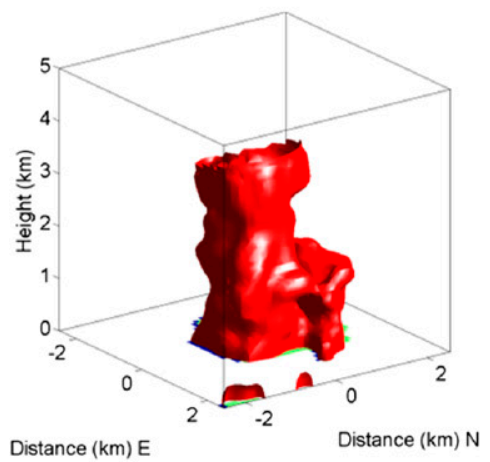

2106:10

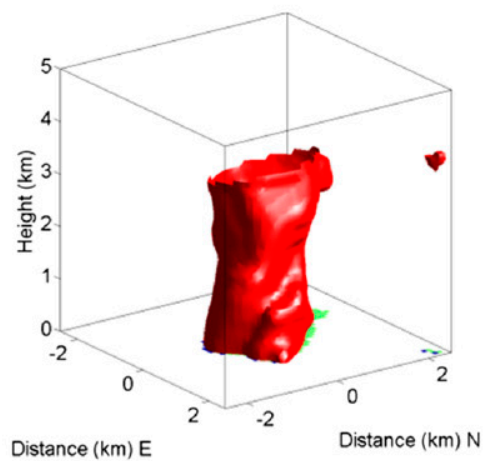

2106:59

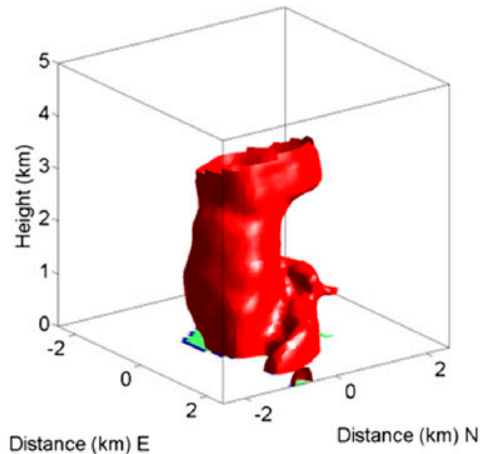

2107:48

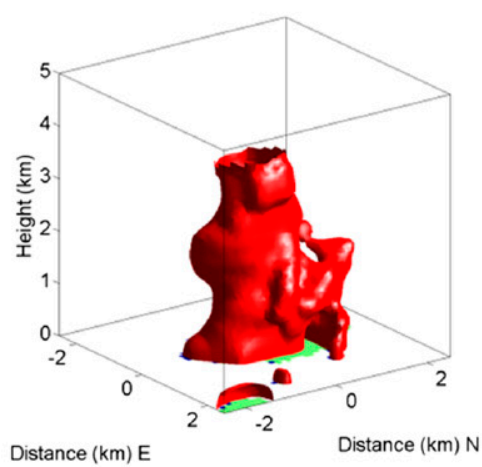

2106:27

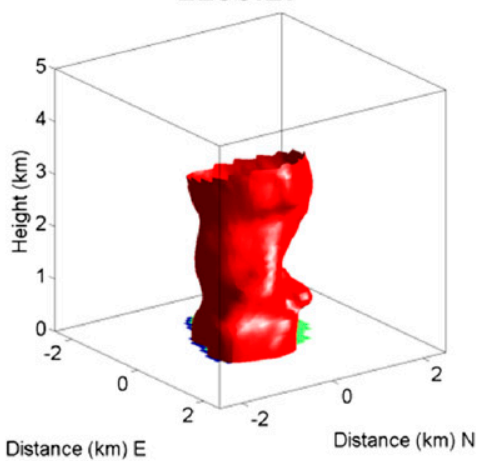

2107:16

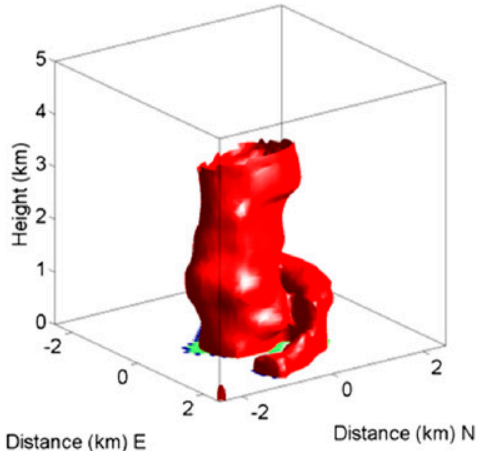

2108:05

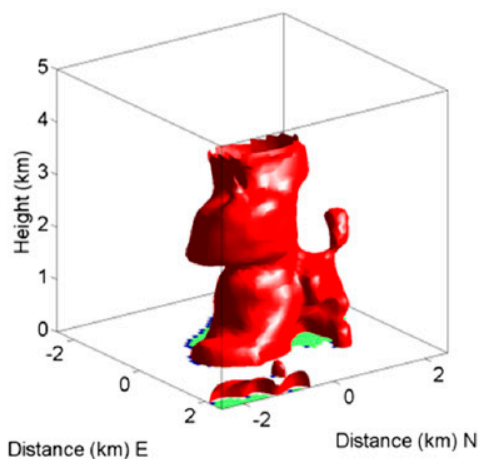

FIG. 10. Three-dimensional (north-south axis on the right, north is directed to the right; east-west axis on the left, west is directed to the left; vertical distance on $y$ axis) isosurfaces of $\rho_{\mathrm{hv}}=0.8$ as viewed from the southeast illustrating the sequence of a debris ejection. The time increment between panels is every volume scan, or about $16 \mathrm{~s}$.

the average $\Delta V_{\max }$ remained relatively constant with time (Fig. 3c), implying that this debris ejection may not have been associated with substantial changes in the internal dynamics of the tornado. Several additional debris ejections occurred after this (e.g., at 2108, 2112, and 2115 UTC, not shown), but beam blockage to the east of the tornado made it difficult to examine the evolution of these ejections.

\section{c. Relationships between the TDS, WEC, and $V_{r}$}

The WEC is a vertical manifestation of the weak echo (reflectivity) hole in the vicinity of a tornado due to a combination of centrifuging effects and subsidence within the core of the tornado (Tanamachi et al. 2012). The WEC associated with this tornado first became evident at 2051:36 UTC, about 15-30s after tornadogenesis 

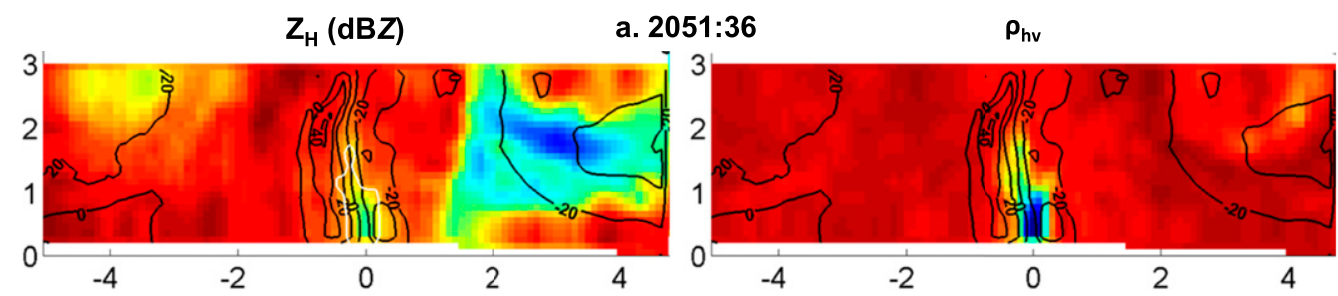

b. $2052: 37$
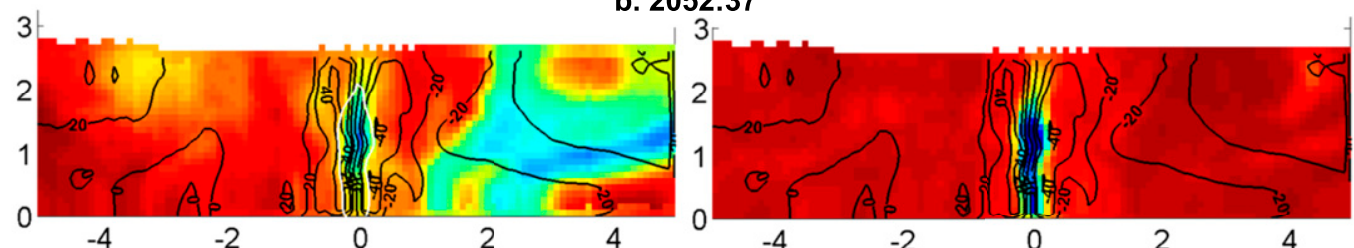

c. $2053: 42$
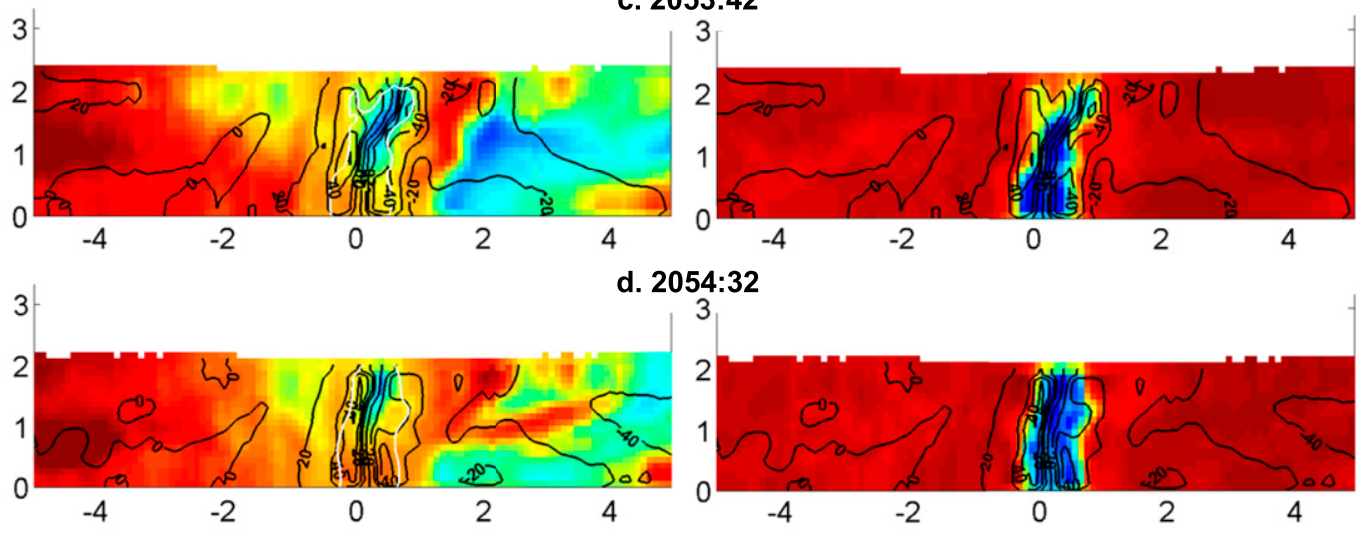

d. 2054:32

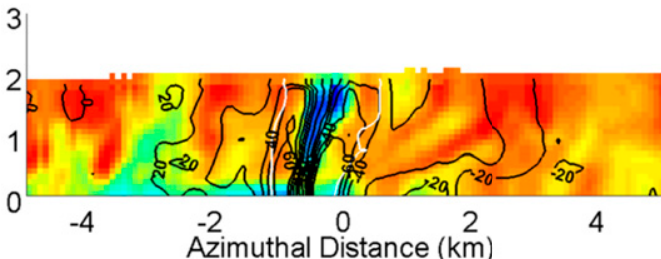

e. 2102:21
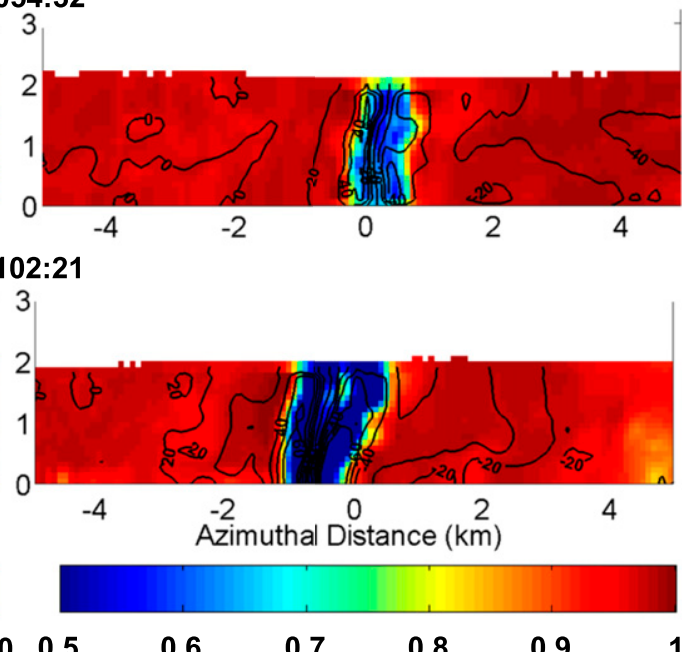

FIG. 11. Vertical cross sections ( $y$ axis is height in $\mathrm{km} \mathrm{ARL)} \mathrm{at} \mathrm{constant} \mathrm{range} \mathrm{(km,} x$ axis) from (left) the radar of the WEC, manifest in the reflectivity field and (right) the TDS, manifest in the $\rho_{\mathrm{hv}}$ field for the beginning of the El Reno tornado: (a) 2051:36, (b) 2052:37, (c) 2053:42, (d) 2054:32, and (e) 2102:21 UTC. Storm-relative Doppler velocity is contoured every $10 \mathrm{~m} \mathrm{~s}^{-1}$ for velocities greater than $20 \mathrm{~m} \mathrm{~s}^{-1}$. The $\rho_{\mathrm{hv}}=0.8$ contour is indicated in white in the reflectivity panels. Data are objectively analyzed and data from the lowest elevation angle are interpolated downward as a function of the critical radius in the vertical $(500 \mathrm{~m})$. The actual heights of the beam at the lowest elevation angle are as follows: (a) 550, (b) 500, (c) 400, (d) 300, and (e) $90 \mathrm{~m}$.

(Fig. 11a). At this time, the shape and the spatial extent of the WEC (defined by the region within the tornado bound by $Z_{H}<30 \mathrm{~dB} Z$ ) were very similar to the TDS visible in $\rho_{\mathrm{hv}}$ bounded by the 0.8 contour. Both were on the order of $300 \mathrm{~m}$ wide and extended to a height of about $1.75 \mathrm{~km}$. The reduction in $Z_{H}$ within the WEC was also visible down to the lowest height at which data were collected (about $300 \mathrm{~m}$ ARL). As the tornado intensified over the next minute, the WEC and TDS grew vertically, extending to a height just over $2 \mathrm{~km} \mathrm{ARL}$, and $Z_{H}$ decreased to $<10 \mathrm{~dB} Z$ (Fig. 11b). The lowest $Z_{H}$ occurred at heights above $1 \mathrm{~km}$, as vertical motions and the cumulative effects of centrifuging on hydrometeors presumably increased with height, while $Z_{H}$ at the lowest heights 
increased by $5-10 \mathrm{~dB} Z$ probably as a combined result of the lofting of larger/more debris near the ground and convergence of hydrometeors and debris in the tornado's near-ground inflow. The shape and spatial extent of the WEC and the TDS still closely matched one another; the TDS was slightly wider than the WEC and both were confined to the width of the radius of maximum wind, which was also the lateral extent of the $40 \mathrm{~m} \mathrm{~s}^{-1}$ isodop.

By 2054 UTC, a notable transition had occurred in the horizontal extent of the TDS, as it became wider, first over the lowest several hundred meters then aloft, presumably as a result of strengthening velocities and an expanding area of tornadic-strength winds. The TDS was no longer the same size as the WEC but extended past the radius of maximum wind and was more closely outlined by the $30-40 \mathrm{~m} \mathrm{~s}^{-1}$ isodop contours (Fig. 11c). At the lowest heights, $Z_{H}$ continued to increase and the lower extent of the WEC was around $750 \mathrm{~m}$ ARL. Within the WEC, low $Z_{H}$ still remained confined to the tornado's core radius, even when the vortex tilted with height. When volume scans resumed at 2102 UTC, the TDS remained wider than the WEC over the depth of the sampling domain (Fig. 11e). The WEC was narrow, having a maximum width of $\sim 700 \mathrm{~m}$ at the top of the domain, whereas the TDS had become considerably wider, having a maximum width of $\sim 1.6 \mathrm{~km}$ aloft. The two features both extended through the top of the sample domain, but the lower extent of the lower boundary of each was different; the TDS extended to the bottom of the sample domain $(<100 \mathrm{~m}$ ARL) while the WEC was only observed to a lower height of $\sim 750 \mathrm{~m}$.

Several minutes later ( 2107 UTC), the TDS became less cylindrical owing to debris shedding and undulations along its outer edge, and the relationship between the boundary of the TDS and the Doppler velocity field became more complex and difficult to interpret (Fig. 12a). However, the TDS was still loosely outlined by the $40 \mathrm{~m} \mathrm{~s}^{-1}$ isodop, except toward the top $1 \mathrm{~km}$ of the domain, where it was bound by weaker Doppler velocities. During times when there were fewer asymmetries in the TDS, the relationship between the TDS and the $40 \mathrm{~ms}^{-1}$ contour appeared more consistent (Figs. 12b,c). When the period of debris fallout was observed, the relationship between Doppler velocity and $\rho_{\mathrm{hv}}$ broke down: as debris fell out of the air from above, it fell into regions with Doppler velocities considerably less than $40 \mathrm{~m} \mathrm{~s}^{-1}$ (Fig. 12d). The WEC remained confined to the region between the tornado's central axis and the radius of maximum wind, regardless of the maximum wind speeds. The shape of the WEC very closely aligned with the orientation of the Doppler velocity contours, indicating a direct relationship between the vertical distribution of the quasi-horizontal wind field and the WEC; where the Doppler velocity contours tilted, so did the WEC.

\section{d. Weak-echo reflectivity band/horizontal vortex}

\section{1) REFLECTIVITY AND VELOCITY OBSERVATIONS OF THE WRB}

As the tornado intensified between 2055 and 2057 UTC, a very narrow [i.e., $O(100) \mathrm{m}$ ] band of cyclonically curved low $Z_{H}$, referred to herein as the WRB, developed immediately to the east of the circulation center and TDS, then wrapped around into the tornado and expanded to its south with time (Fig. 13). By 2056:53 UTC, the feature was well defined (Fig. 13g) as a region of $Z_{H}$ deficits of $\sim 15-30 \mathrm{~dB} Z$ relative to the surrounding area. The greatest $Z_{H}$ deficit of $35 \mathrm{~dB} Z$ was observed near the center of this feature and was achieved at 2057: 15 UTC. The WRB became more elongated through about 2058 UTC (Fig. 13k) and afterward became narrower and disconnected from the tornado's TVS. From animations it is seen that elements within the reflectivity pattern along the periphery of the WRB circulated into the tornado (see the online supplemental material; WRB animation). By 2058:07 UTC, the only evidence of the WRB was the narrow trailing line of slightly reduced $Z_{H}$ southeast of the tornado (Fig. 131).

Another radar, the mobile weather radar $2005 \mathrm{X}$-band phased-array (MWR-05XP; Bluestein et al. 2010) simultaneously collected volumetric data on this tornado between 2050 and 2056 UTC (French et al. 2014), undeploying less than one minute prior to when the WRB became evident in the RaXPol data. A single-elevation ${ }^{6}$ dual-Doppler analysis was synthesized at 2056:08 UTC in an effort to examine the WRB's correlation with the near-surface kinematic wind field (Fig. 14). Although the dual-Doppler horizontal winds likely contain quantitative errors owing to the complexities discussed in the appendix, they provide a qualitative overview of the kinematics in the vicinity of the WRB.

The WRB formed within a zone of convergence associated with a secondary RFGF surge (Figs. 14a,e). The leading edge of the primary RFGF was several hundred meters to the east of the leading edge of a secondary RFGF surge. The mature WRB was collocated with perturbations in the Doppler velocity field (Fig. 15). By 2056:53 UTC, the southern portion of the WRB was

\footnotetext{
${ }^{6}$ Only one elevation angle was analyzed because, at this time, RaXPol was operating in rapid-scan "tornado" mode, collecting 2-s data updates at a constant $1^{\circ}$ elevation angle; RaXPol did not collect volumetric data during this period. This scanning strategy also precluded the ability to evaluate the vertical extent, structure, and evolution of the WRB.
} 


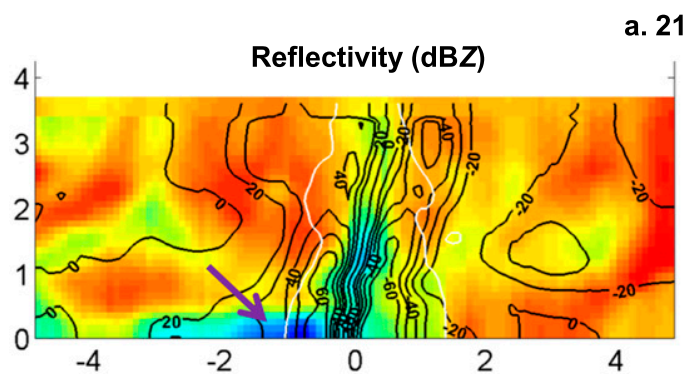

a. $2107: 40$

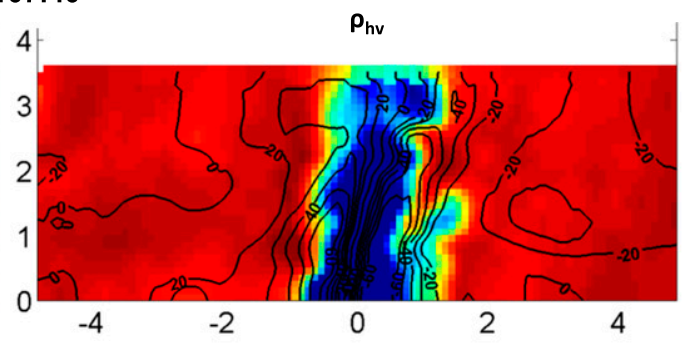

b. 2109:51
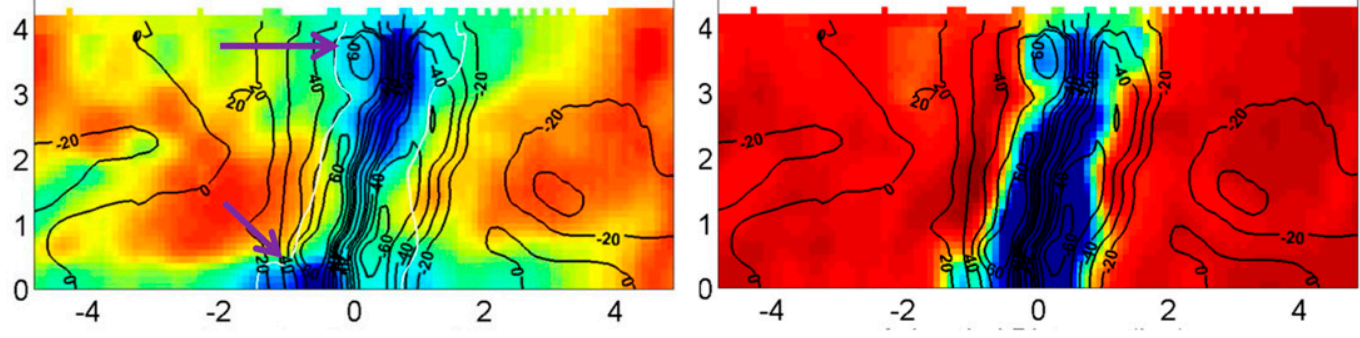

c. $2111: 46$
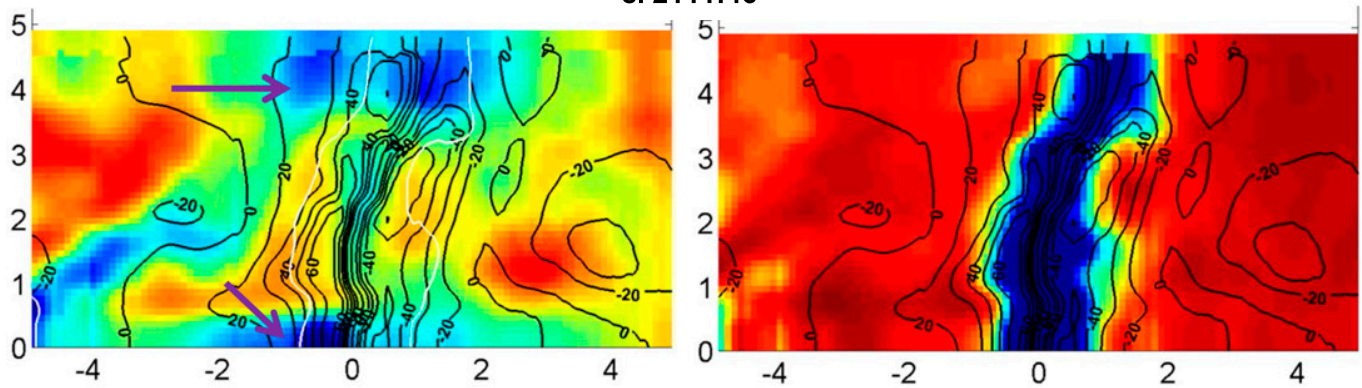

d. 2116:24
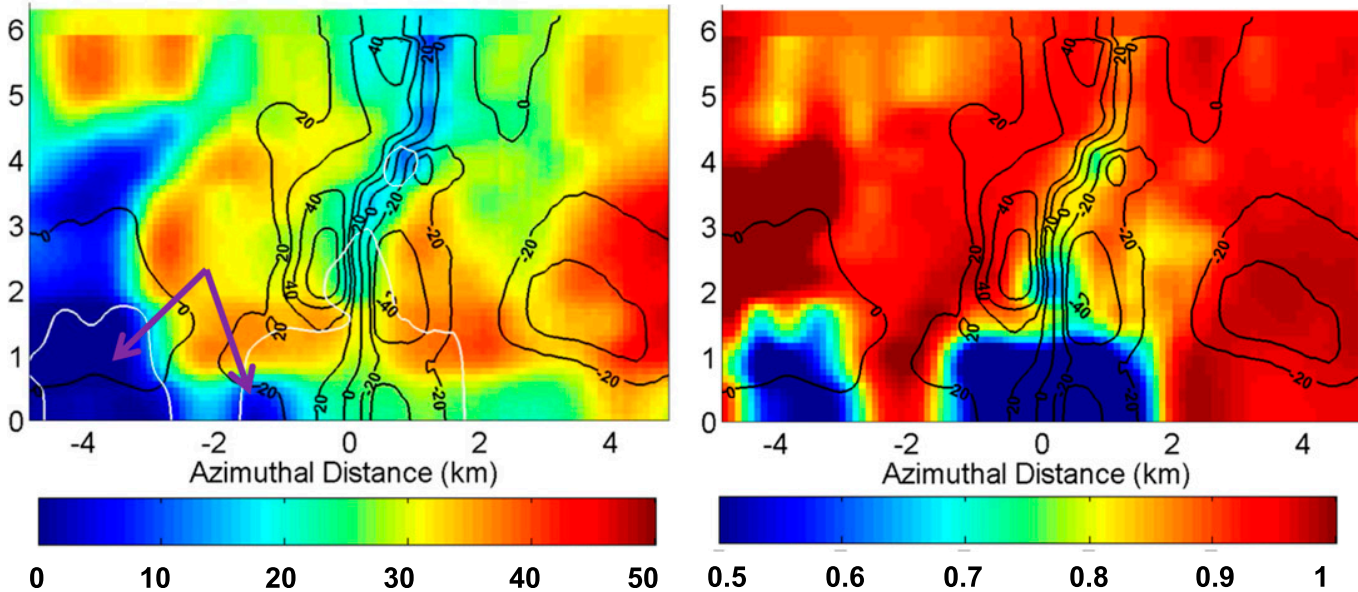

FIG. 12. As in Fig. 11, but for (a) 2107:40, (b) 2109:51, (c) 2111:46, and (d) 2116:24 UTC. Purple arrows point to areas of signal loss due to severe attenuation. Minimum beam heights for these figures are (a) 170, (b) 210, (c) 200, and (d) $320 \mathrm{~m}$.

collocated with a narrow band of inbound (flow with a southerly component) Doppler velocities, straddled by two regions of outbound velocities (flow with a northerly component) (Fig. 15g). The region of strong inbound velocities nearly perfectly matched the width of the reflectivity trough. There was radial divergence along the leading edge of the feature and radial convergence just behind it (Figs. 16a,b), similar to what was observed earlier, in the dual-Doppler analyses. Such a configuration of horizontal winds at near-ground level implies 

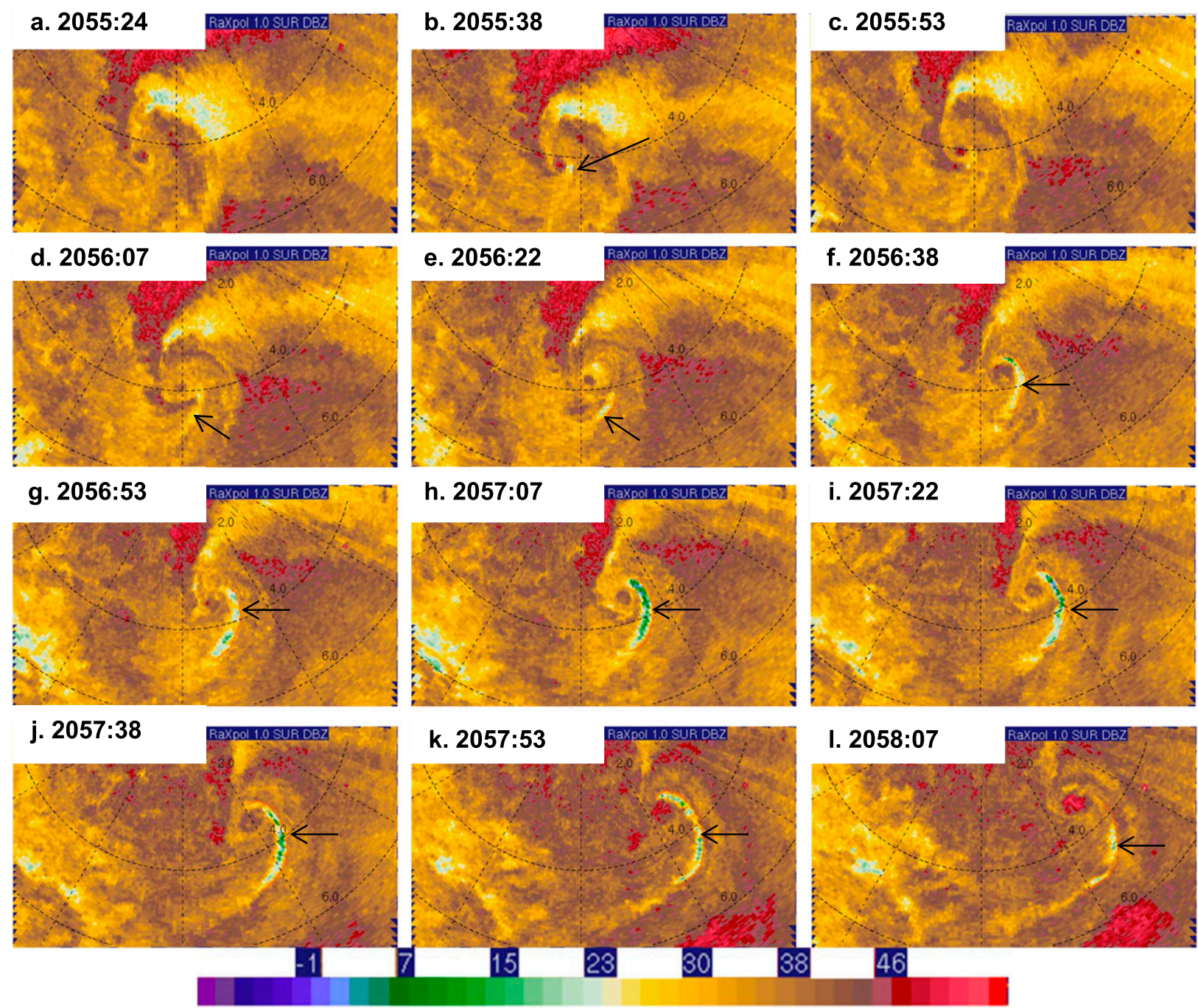

FIG. 13. Reflectivity images from the $1^{\circ}$ elevation angle depicting the development of the WRB at $\sim 15$-s increments from (a) 2055:24 to (l) 2058:07 UTC. Range rings are every $2 \mathrm{~km}$. The arrow points to the location of the WRB.

a downdraft along the leading (southeastern) edge of the feature, and upward motion on the back (northwestern) side consistent with a horizontal vortex.

The trailing edge of the WRB was collocated with cyclonic misovortex signatures, similar to those observed along the primary RFGF by Bluestein et al. (2003, their Fig. 10), Lee et al. (2012), and Wurman and Kosiba (2013) (Fig. 16). It is unclear why these misovortices formed, but since the gradient in Doppler velocities was large and there was horizontal wind shear apparent from the dual-Doppler analysis along the interface of the secondary RFGF (Fig. 14), it is possible that they formed due to shearing instability. As the WRB began to decay (Figs. 13i,j), the channel of strong inbound Doppler velocities became narrower and less continuous (Figs. 15i,j). The weakening trend continued as the WRB wrapped around the tornado, and it decayed entirely by 2059 UTC (not shown).

\section{2) Polarimetric ObSERVATIONS OF THE WRB}

The WRB was associated with slightly reduced $\rho_{\mathrm{hv}}$ $(\sim 0.85-0.9)^{7}$ and low $Z_{\mathrm{DR}}(-1-0 \mathrm{~dB})$. When the WRB became obvious in $Z_{H}$, a small sawtooth feature was

\footnotetext{
${ }^{7}$ Because $Z_{H}$ decreased below $20 \mathrm{~dB} Z$, a portion of the reduction in $Z_{\mathrm{DR}}$ and $\rho_{\mathrm{hv}}$ may be due to a decrease in signal-to-noise ratio (SNR), which negatively biases both these polarimetric parameters. However, the SNR remains generally $>20-25 \mathrm{~dB}$, which is typically sufficient for reliable $Z_{\mathrm{DR}}$ and $\rho_{\mathrm{hv}}$ retrievals. Additionally, there was comparably low $Z_{H}$ at similar ranges, which was not associated with similarly reduced $\rho_{\mathrm{hv}}$ and $Z_{\mathrm{DR}}$. Therefore, the decrease in these parameters is likely not a result of low SNR bias.
} 


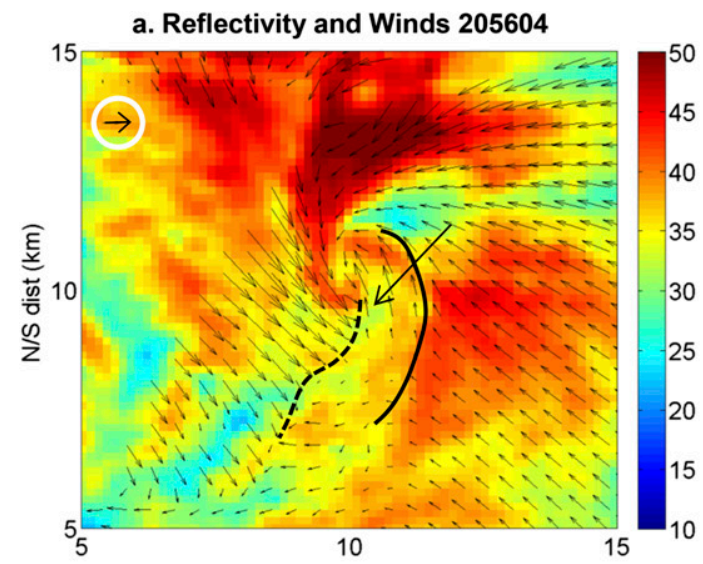

\section{b. Radial Velocity and Winds 205604}

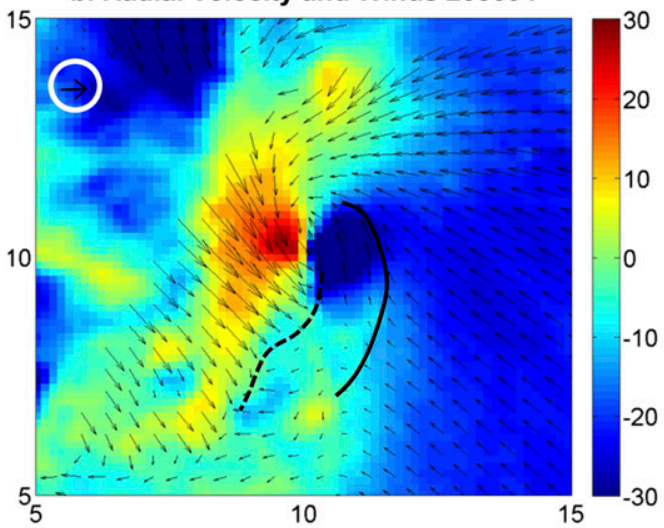

d. $\rho_{\text {hv }}$ and Winds 205604

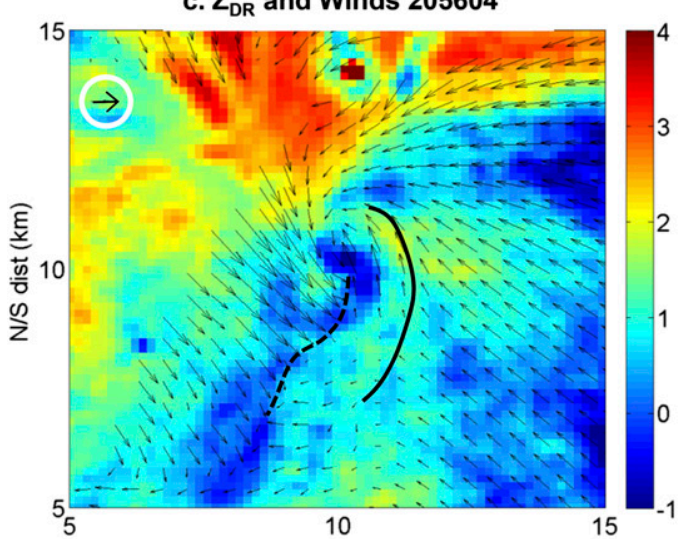

e. Divergence and Winds 205604

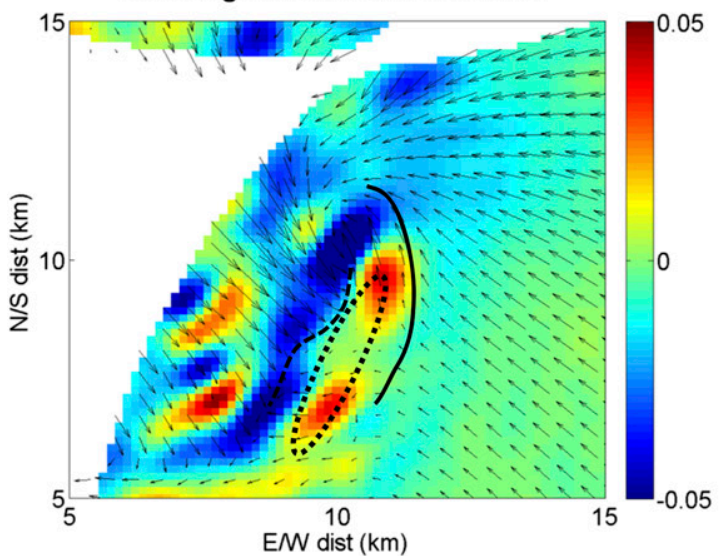

FIG. 14. Storm-relative horizontal velocity vectors synthesized from a single-elevation dual-Doppler analysis from RaXPol and MWR-05XP valid at 2056:04 UTC for $z=250 \mathrm{~m}$ : (a) reflectivity, (b) $\rho_{\mathrm{hv}}$, (c) $Z_{\mathrm{DR}}$, (d) RaXPol storm-relative Doppler velocity, and (e) horizontal divergence $\left(\times 10^{-3} \mathrm{~s}^{-1}\right)$ (warm colors indicate divergence, cold colors convergence). Reference vector circled in white is $25 \mathrm{~m} \mathrm{~s}^{-1}$. The solid (dashed) black curve indicates the leading edge of the primary (secondary) RFGF. The black arrow in (a) denotes the location where the weak-echo reflectivity band developed over the next minute. The dotted black circle in (e) indicates a region of divergence possibly associated with an occlusion downdraft.

apparent in $\rho_{\mathrm{hv}}$ (Fig. 17). There also was a band of slightly reduced $\rho_{\mathrm{hv}}$ (e.g., $\sim 0.85-0.9$ ) immediately behind the leading edge of the WRB, which appeared to be residual debris from an earlier debris ejection feature.
As the WRB persisted and $Z_{H}$ decreased, low $\rho_{\mathrm{hv}}$ became more evident in the vicinity of the WRB, implying that there was likely some nonmeteorological debris present where $\rho_{\mathrm{hv}}$ was reduced. It is important to note 

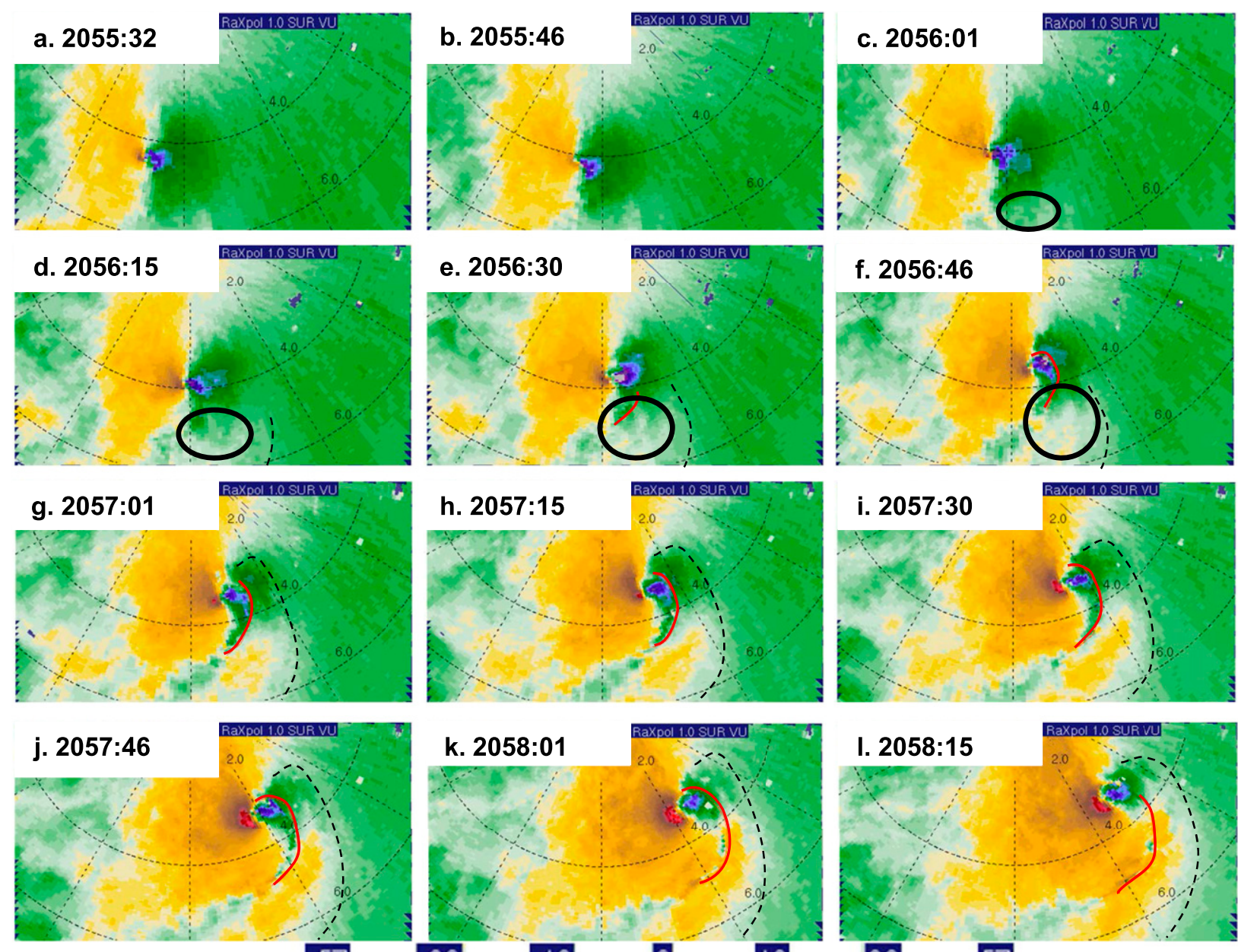

$-57$

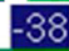

$-19$

0

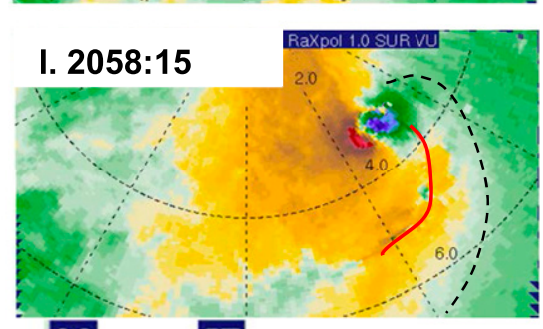

38

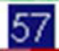

FIG. 15. As in Fig. 13, but for Doppler velocity $\left(\mathrm{m} \mathrm{s}^{-1}\right)$. The red curve indicates the location of the leading edge of the WRB, the black dashed curve indicates the most likely location of the primary RFGF, and the black ellipse denotes the region east of the secondary RFGF, where the divergent flow develops and the sign of $V_{R}$ changes with time.

that the reduced $\rho_{\text {hv }}$ was not associated with a debris ejection and $\rho_{\mathrm{hv}}$ was considerably higher and reduced over a narrower channel than they were within the previously discussed debris ejections. The higher $\rho_{\mathrm{hv}}$ coupled with lower values of $Z_{H}$ suggest that if debris were indeed present, it was likely either smaller or lower in concentration than the debris associated with the debris ejections. A separate band of reduced $\rho_{\mathrm{hv}}$ also developed ahead of the one associated with the $\mathrm{WRB}$, and a debris ejection with greatly reduced $\rho_{\mathrm{hv}}$ was observed toward the end of the WRB's life, emphasizing the relatively inconspicuous nature of the $\rho_{\text {hv }}$ field in the vicinity of the WRB (Figs. $17 \mathrm{~g}-1$ ).

The WRB was also collocated with a region of reduced $Z_{\mathrm{DR}}$ (Fig. 18). Around $2056 \mathrm{UTC}$, a tail of reduced $Z_{\mathrm{DR}}$ bound to the east by a band of slightly higher $Z_{\mathrm{DR}}(1-2 \mathrm{~dB})$ collocated with the decreasing $Z_{H}$ became apparent
(Fig. 18). The spatial relationship between the $Z_{H}$ trough and the lower $Z_{\mathrm{DR}}$ (consistently between -1 and $\left.0 \mathrm{~dB}\right)^{8}$ persisted as the band became more pronounced in both fields. The $Z_{\mathrm{DR}}$ depression was generally longer than the WRB, extending farther $S W$. As the WRB decayed, $Z_{\mathrm{DR}}$ within the WRB increased. The juxtaposition of the WRB with the local reductions in $Z_{\mathrm{DR}}$ and $\rho_{\mathrm{hv}}$ indicates that this region was most likely composed of a low number concentration of small raindrops, perhaps mixed with hail and/or small nonmeteorological debris such as dust and sand to account for the reduction in $\rho_{\mathrm{hv}}$.

\footnotetext{
${ }^{8}$ At this time, moderate-to-heavy rain was falling at the radar deployment location. Because the radome was wet, attenuation and differential attenuation likely were significant, though difficult to quantify.
} 

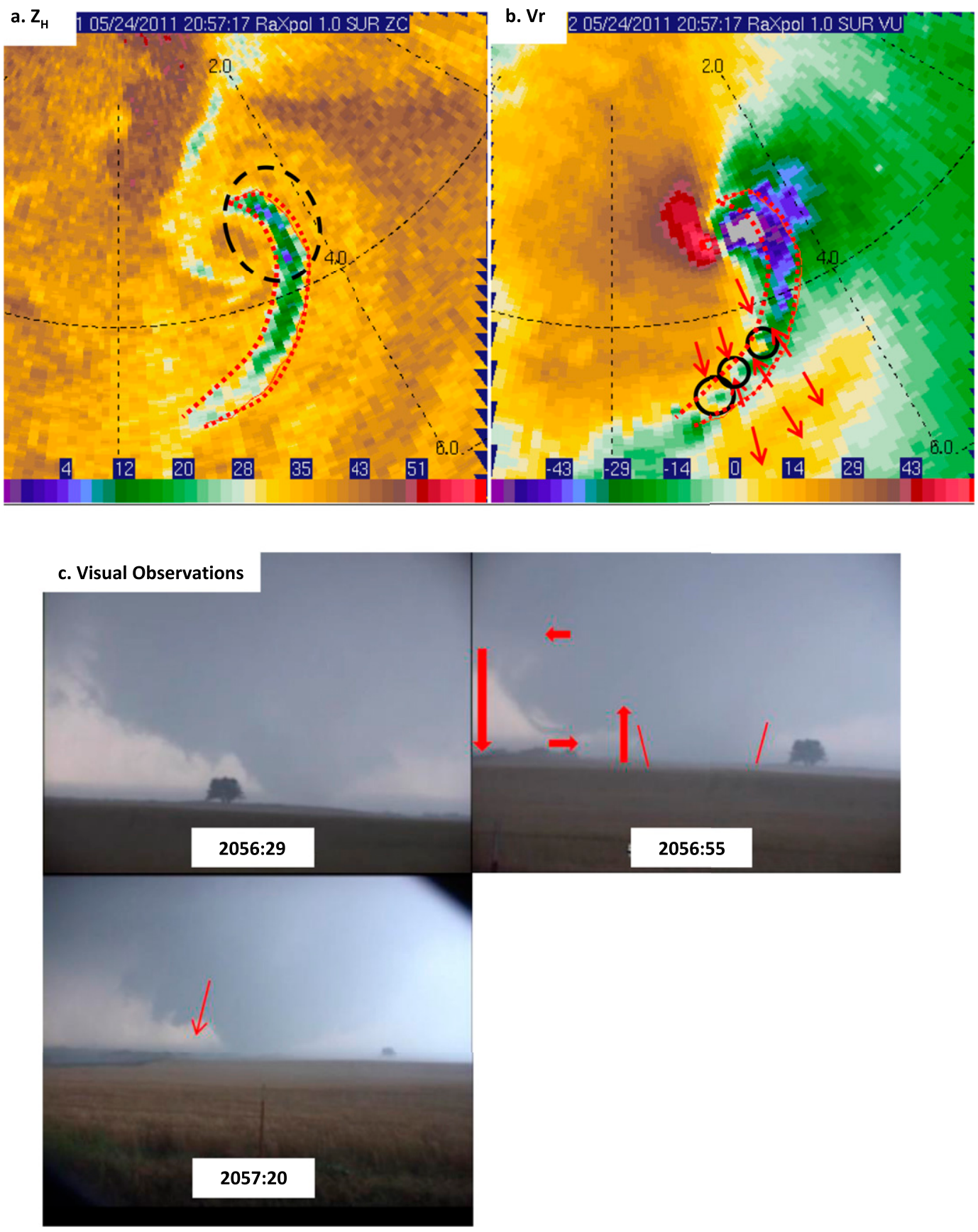

FIG. 16. Zoomed-in image of the (a) reflectivity and (b) radial velocity fields associated with the WRB from $1^{\circ}$ elevation ( $\sim 60 \mathrm{~m}$ ARL) at 2057:35 UTC. The red dashed curves outline the WRB, the solid black circle denotes the misocyclones at the end of the WRB, the dashed black circle indicates the portion of the WRB observed by the radar crew, and the red arrows indicate relative Doppler flow across the band, indicating low-level divergence along the leading edge and convergence along the trailing edge of the feature. (c) A series of visual images of the horizontal vortex and observed vertical motions as observed from the north of the tornado. The condensation funnel associated with the tornado is outlined in red for visual clarity.

\section{3) HORIZONTAL VORTEX}

Coincident with the formation and location of the WRB, the radar crew visually observed a rapidly rotating horizontal vortex to the east of the tornado from their deployment location several kilometers north of the tornado (Fig. 16c) and the two features are believed to be the same (Fig. 15a). The visually observed vortex was 

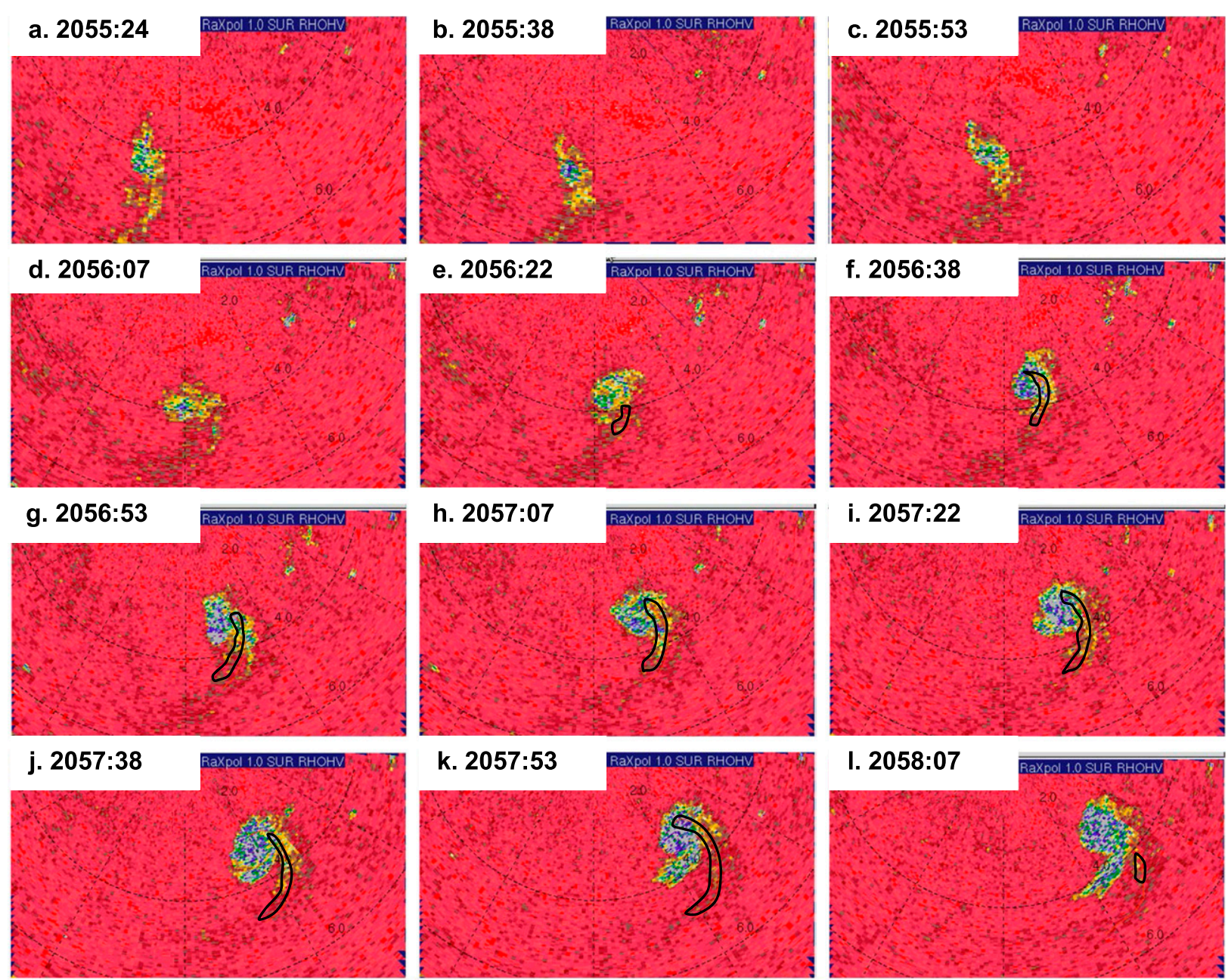

I. 2058:07
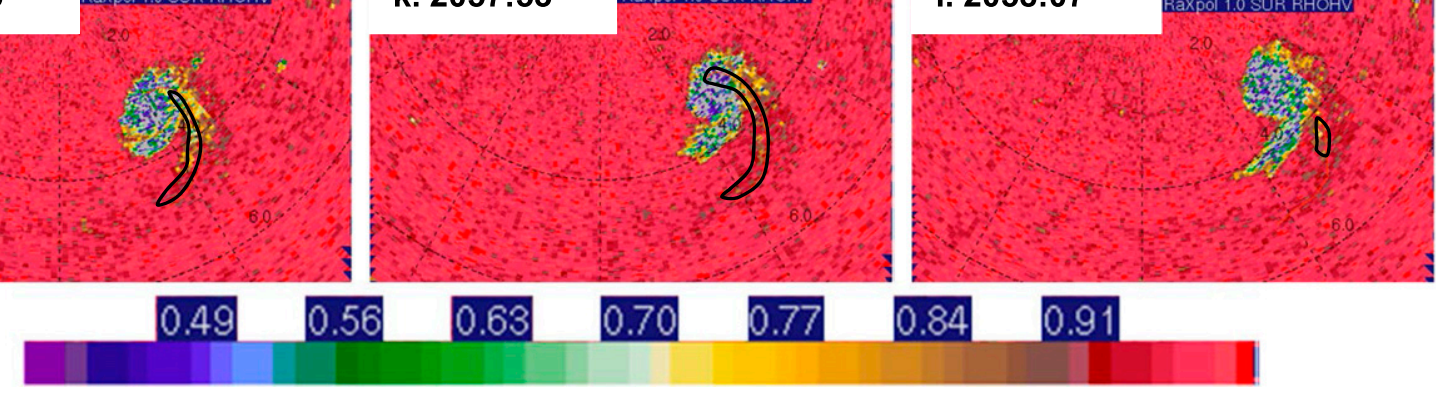

FIG. 17. As in Fig. 13, but for $\rho_{\text {hv }}$. Black outline traces the WRB.

$\sim 750 \mathrm{~m}$ wide while the WRB was $\sim 250 \mathrm{~m}$ wide, implying that there was precipitation within the periphery of the horizontal vortex. A rapid sinking motion was observed on the eastern or leading side of the horizontal vortex, and upward motion was evident on the western or trailing side. Thus, the vorticity vector was directed toward the north or northeast. This observation is consistent with the qualitative analysis of vertical motion implied from the zones of lowlevel divergence and convergence noted in the Doppler velocity data. Wurman and Kosiba (2013) observed horizontal vortices in the same place relative to the tornado as the one observed herein, but did not mention the vortices being collocated with a feature like the WRB. Bluestein et al. (2007) also observed a horizontal vortex near a tornado.

Over the 2-min period during which the WRB was observed, the maximum difference between inbound and outbound radial velocities $\left(\Delta V_{\max }\right)$ associated with the tornado increased from $\sim 120$ to $180 \mathrm{~m} \mathrm{~s}^{-1}$, then settled to a nearly constant $\sim 150 \mathrm{~m} \mathrm{~s}^{-1}$ (Fig. 3b). The time during which the $\Delta V_{\max }$ increased the most rapidly is coincident with the time when the horizontal vortex and WRB were developing and intensifying ( $\sim 2055: 40$ to $\sim 2057: 00$ UTC). According to both the radar data and visual observations, the WRB and the horizontal vortex both appeared to wrap into the tornado. After $\sim$ 2057:00 UTC, the intense horizontal vortex dissipated, although it became visually obscured by rain so the exact time of dissipation is unknown. The approximate time when the horizontal vortex decayed was also coincident with the cessation in intensification of $\Delta V_{\max }$. Thus, there appears to be a relationship between the intensification of the tornado and the appearance of the horizontal vortex/ WRB. Unfortunately, based upon the data that are available, it is unclear whether the horizontal vortex contributed 

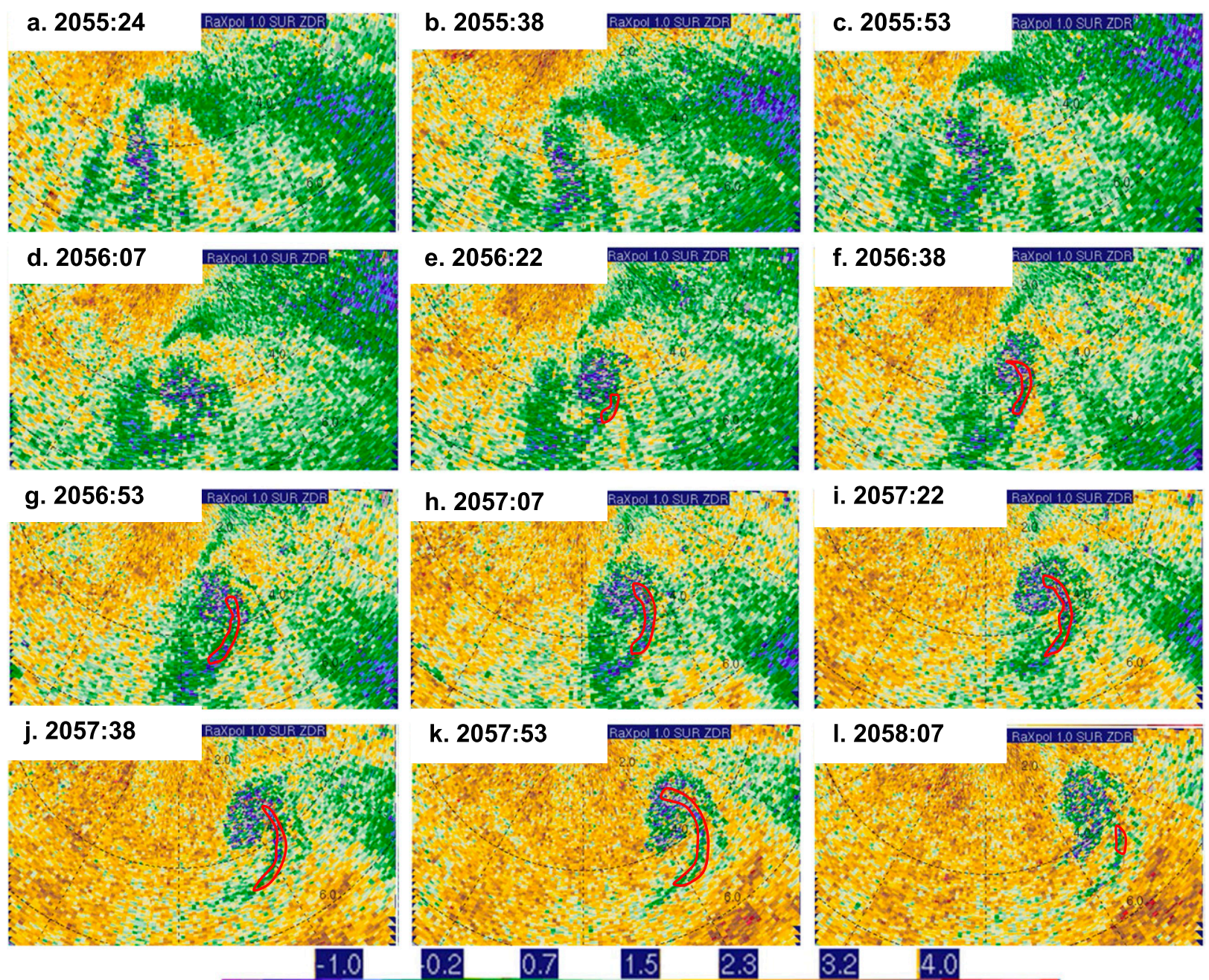

FIG. 18. As in Fig. 17, but for $Z_{\mathrm{DR}}(\mathrm{dB})$.

to the intensification of the tornado via tilting and stretching of horizontal vorticity, or if the horizontal vortex was a result of strengthening inflow into the tornado, stretching ambient horizontal vorticity in a localized inflow channel.

\section{4) Possible formation MeCHANisms}

It is suspected from the radar data and visual observations that the WRB/horizontal vortex most likely developed at low levels; although the precise formative causes of it are unclear based upon the available observations alone. The discussion that follows is mostly speculative, and uses the best information available given the data collected. Nonetheless, because there has been minimal discussion in the literature explaining why such a feature may form, we will present several hypotheses describing some possible mechanisms that may have aided in the formation of the feature. We will not address all the myriad ways that horizontal vorticity could be generated in the context of this feature.

The horizontal vorticity equation under the Boussinesq approximation can be written as follows:

$$
\begin{aligned}
\partial \boldsymbol{\omega}_{h} / \partial t= & -\mathbf{v} \cdot \nabla \boldsymbol{\omega}_{h}+(\boldsymbol{\omega}+f \mathbf{k}) \cdot \nabla \mathbf{v}_{h} \\
& +\nabla \times \mathbf{B k}+\nabla_{h} \times \mathbf{F},
\end{aligned}
$$

where $\boldsymbol{\omega}_{h}$ is the horizontal vorticity vector, $f$ is the Coriolis parameter, $\mathbf{B}$ is buoyancy, and $\mathbf{F}$ is friction. According to this equation, horizontal vorticity can only be generated baroclinically or frictionally, though preexisting vertical vorticity can be tilted into the horizontal. Baroclinically generated horizontal vorticity can form along a thermal (density) gradient, for example between the RFD and the environmental air. In this case, the WRB and horizontal vortex formed behind the 
leading edge of the RFGF, in the vicinity of a secondary RFGF, in a similar location to a region of enhanced horizontal vorticity derived from a horizontal vorticity budget by Kosiba et al. (2013). Unfortunately, there was no way to retrieve the horizontal wind components at the time the vortex was well developed. Additionally, the presence of the vortex modified the ambient wind field. Therefore, the evolution of the wind field in the context of the secondary RFGF and the horizontal vortex was unclear, as the two features evolved simultaneously and were not independent of each other. The nebulous evolution and placement of the RFGF with respect to the horizontal vortex precluded a clear determination of whether the horizontal vortex formed along, immediately ahead of, or immediately behind the secondary RFGF.

If the vortex formed as a result of baroclinically generated horizontal vorticity associated with the thermal gradient between the air ahead of and behind the secondary RFGF, then, in order to match the observed orientation of the horizontal vorticity vector, the air behind the secondary RFGF must have been warmer than that behind the primary RFGF. Several studies have supported the notion that warmer air can exist behind the secondary RFGF than behind the primary one (e.g., Grzych et al. 2007; Hirth et al. 2008; Lee et al. 2012; Marquis et al. 2012). While thermodynamic observations were not available to verify the temperature perturbation behind the secondary RFGF, this is one potential mechanism by which the horizontal vortex was created: the generation of horizontal baroclinic vorticity along the secondary RFGF boundary with relatively warm air (relative to the air behind the primary RFGF) located behind the secondary RFGF (Fig. 19a).

Another possible explanation for the formation of the vortex/WRB is that it was generated through frictional processes, as suggested by some numerical studies (Lewellen et al. 2000; Schenkman et al. 2014), either due to the preferential ingestion of low angular momentum inflow into the tornado as a result of friction and the translation of the vortex (Lewellen et al. 2000) or by friction acting directly on the low-level inflow or outflow, generating horizontal vorticity (Schenkman et al.2014). Schenkman et al. (2014) identified frictional generation of horizontal vorticity in the outflow behind the RFGF, in a similar location to what was observed in this case. According to the dual-Doppler analysis, flow behind the secondary RFGF was northwesterly. If it is assumed that flow very near the surface is slower than flow several hundred meters aloft, the component of frictionally generated vorticity associated with the vertical wind shear would be directed to the northeast, in agreement with the observed orientation of horizontal

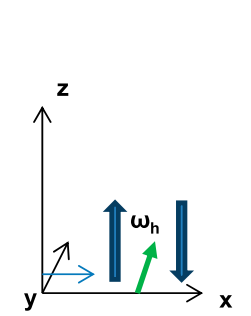

a. Baroclinic generation

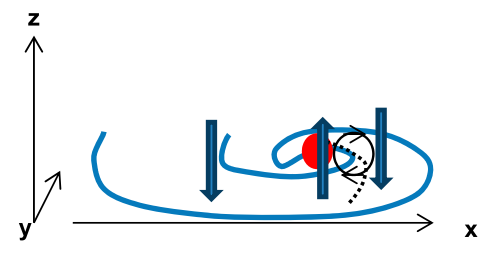

b. Frictional generation
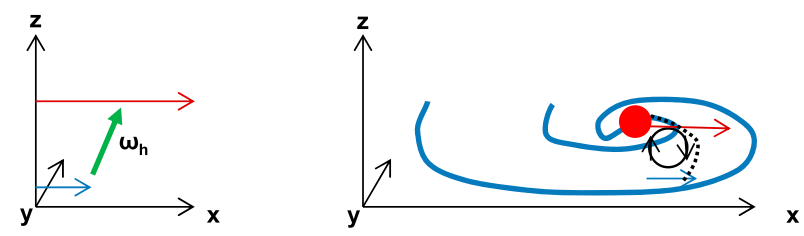

c. Stretching of the horizontal vorticity

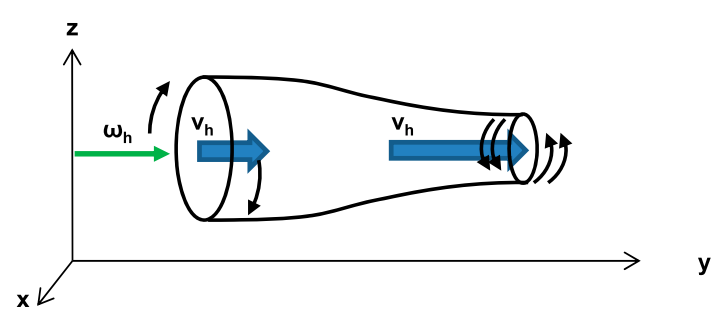

FIG. 19. Conceptual figure illustrating the possible mechanisms of forming the horizontal vortex and WRB. (a) Baroclinically generated vorticity between warmer air behind the secondary RFGF and cooler air behind the primary RFGF. (b) Frictionally generated horizontal vorticity by the secondary RFGF. (c) Stretching of the horizontal vorticity by strengthening inflow. Near-surface horizontal winds are indicated by light blue arrows while low-level $(z \sim 200 \mathrm{~m})$ winds are indicated by maroon arrows, vertical winds by wide dark blue arrows, green arrow indicates direction of horizontal vorticity vector, black circle and arrows denote rotational sense of vorticity, red dot indicates tornado location, and black dashed line indicates the secondary RFGF. The bold blue outline denotes the $25-\mathrm{dB} Z$ contour near the surface.

vorticity. Such a mechanism would result in a vortex behind the secondary RFGF (Fig. 19b).

A final possibility is that vertical vorticity associated with the near-tornado environment, or with the tornado itself, could have been tilted into the horizontal (not shown). Regardless of its source, the horizontal vorticity present in the vicinity of the secondary RFGF was most likely stretched by increased inflow into the strengthening tornado, forming the horizontal vortex along the secondary RFGF (Fig. 19c).

The reason for the deficit in $Z_{H}$ associated with the WRB is also unclear. The most likely reason is that centrifuging within the horizontal vortex evacuated the hydrometeors from the area. This hypothesis is bolstered by the observations that the WRB was located between 
the convergence and divergence zones, rather than centered on one or the other. Since the $Z_{H}$ deficit increased with increasing convergence/divergence, stronger horizontal vorticity, presumably the result of stretching by strong inflow may have been associated with a reduction of hydrometeors. It is possible, however, that either the upward motion on the western flank of the horizontal vortex lofted falling hydrometeors upward, preventing rain from falling through the updraft, or a dynamically driven occlusion downdraft to the east of the tornado contributed to a reduction in hydrometeors, in a manner similar to how a clear slot develops. Since the available observations preclude the ability to resolve vertical motions, neither of these hypotheses can be substantiated.

\section{Discussion and conclusions}

This study examined various structural and polarimetric aspects of the TDS and a WRB associated with a violent tornado that occurred on 24 May 2011. It was determined that the TDS formed when the low-level $\Delta V_{\max }$ exceeded $50 \mathrm{~m} \mathrm{~s}^{-1}$, despite the absence of a vertically coherent vortex. Its formation was aided by the presence of remnant nonmeteorological debris present from a previous tornado, and from debris accumulation along the RFGF that was concentrated in the low-level mesocyclone and developing tornado vortex. The TDS quickly grew to a height that exceeded the top of the radar domain and was observed at heights above $5 \mathrm{~km}$ ARL when the tornado reached its mature state. Several distinct heterogeneities within the TDS were observed including the following: 1) wavelike oscillations that propagated upward along the outer edge of the TDS; 2 ) small-scale $[\sim O(100) \mathrm{m}]$ sawtooth-like features hypothesized to be associated with lofted debris from spiral inflow bands; 3) a substantial widening of the lower portion of the TDS as the tornado weakened, consistent with lofted, suspended debris falling out of the weakening tornadic updraft; and 4) comma-shaped features of low $\rho_{\mathrm{hv}}$ consistent with the debris ejections described by Kurdzo et al. (2015). The debris ejections had three-dimensional structure and extended to heights of about $1 \mathrm{~km}$, becoming higher the closer they were to the main TDS. These events occurred cyclically, some occurring as quickly as $1 \mathrm{~min}$ apart. Several (but not all) of the debris ejections were associated with surges in a secondary RFGF.

The WEC was narrower than the TDS-an observation also noted by Wakimoto et al. (2015) in a different tornado-except for the first several minutes after tornadogenesis. The bottom boundary of the TDS extended to the lowest observed radar level, but the base of the WEC generally terminated at a height $\sim 750 \mathrm{~m}$ ARL except again as the tornado was first forming, when it too extended to the lowest observed level. Both features extended through the top of the radar domain except when the tornado weakened. Based upon the relationships between the Doppler velocity field and the WEC it appears that the actual magnitude of the Doppler velocity did not affect the width of the WEC. Rather, the WEC remained concentrated within the strongest radial velocity gradient between the tornado's central axis and the radius of maximum wind. This relationship between the WEC and the Doppler velocity field is in agreement with the results found in Dowell et al. (2005), who conclude that centrifuging is associated with horizontal and vertical vorticity maxima. The region within the radius of maximum wind, where the gradient in Doppler velocities is strongest, is one such example of a vorticity maximum. Therefore, in this region, hydrometeors and other material including debris will be centrifuged outward, resulting in a relative minimum in $Z_{H}$ because of the relatively low number concentration of scatterers. The tilted structure of the WEC observed herein is consistent with this notion as the centrifuging remains closely associated with the vorticity maximum.

The TDS, conversely, appeared to be sensitive to the actual magnitude of the storm-relative Doppler velocity, often being closely bound near the $40 \mathrm{~m} \mathrm{~s}^{-1}$ isodop on either side of the axis of rotation. This relationship implies that under the conditions of this case study, debris became lofted, forming a vertically coherent TDS that was detectable by a mobile X-band radar only when Doppler velocities exceeded $\sim 40 \mathrm{~m} \mathrm{~s}^{-1}$, or approximately EF-1 intensity. This observation is consistent with what Wakimoto et al. (2012) found in their combined dual-Dopplerphotogrammetric analysis of a strong tornado in Wyoming; however, it is not consistent with some studies that have observed TDSs with weaker (EF-0) tornadoes (e.g., Schultz et al. 2012; Van Den Broeke and Jauernic 2014).

The WRB, a narrow, arcing band of reduced $Z_{H}$ observed in the immediate vicinity of a secondary rear-flank gust front, to the east through south of the tornado, was straddled by a zone of low-level quasi-horizontal divergence to its east and quasi-horizontal convergence to its west. The location of the WRB was strikingly close to that of an observed horizontal vortex visually identified with rising motion on its west and sinking on its east. The horizontal vortex developed as the tornado underwent a period of rapid intensification, and it is hypothesized that the feature was correlated with the intensification, although no causality between the horizontal vortex and the intensification of the tornado could be determined. Bands of reduced $\rho_{\mathrm{hv}}$ and $Z_{\mathrm{DR}}$ were nearly collocated with the WRB. The combination of low $Z_{H}$, low $Z_{\mathrm{DR}}$, and slightly reduced $\rho_{\mathrm{hv}}$ can most likely be explained by the presence of a mixture of a low number concentration of small raindrops and small debris such as sand and dirt. 
A summary of possible mechanisms for generating horizontal vorticity with the orientation observed in this case, and possible configurations of storm-scale and environmental features that are consistent with the visual and radar observations of the horizontal vortex, are presented in Fig. 19. It is proposed that the development of the WRB and horizontal vortex may have formed by one of the following processes: 1) baroclinic generation of horizontal vorticity between the primary and secondary RFGFs, such that the air behind the secondary RFD surge was warmer than that behind the primary RFGF; 2) through frictional processes occurring in the outflow air immediately behind an RFGF surge; or 3) through tilting of vertical vorticity into the horizontal in the vicinity of the tornado or from the tornado itself. Regardless of how the horizontal vorticity was generated, it was most likely further amplified through stretching along the accelerating inflow, which generated progressively stronger vertical motions associated with the ascending and descending branches of the horizontal vortex, and the WRB became more pronounced as centrifuging and/or vertical motions intensified.

It is evident from this study that the observations of the TDS and the WRB were complex and showed considerable variation on short times scales ( $<1 \mathrm{~min})$. The need for multiple rapid-scan radars collecting data simultaneously is evident as dual-Doppler analyses would help to elucidate some of the complexities encountered while trying to explain the kinematic aspects of these features.

Acknowledgments. This work was supported by NSF Grants AGS-0821231 and AGS-0934307 awarded to the second author at the University of Oklahoma. Funding to support the third author was provided by NOAA/ Office of Oceanic and Atmospheric Research under NOAA-University of Oklahoma Cooperative Agreement NA11OAR4320072, U.S. Department of Commerce. The authors additionally acknowledge the contributions of the Advanced Radar Research Center led by Robert Palmer, and particularly John Meier for hardware and software support. David Dowell provided the objective analysis code. Patrick Skinner provided useful comments on an early draft of the manuscript. Finally, the authors thank three anonymous reviewers and Yvette Richardson for their valuable contributions, which improved the quality and accuracy of the manuscript.

\section{APPENDIX}

\section{Objective Analysis and Dual-Doppler Analysis}

Two objective analysis procedures were used to interpolate the radar data into common Cartesian space.
The first procedure was for the single-Doppler RaXPol volumes used to analyze the time-height evolution of the TDS and WEC. For these analyses, a two-pass Barnes's analysis scheme (Barnes 1964) was used to construct grids over a domain of $10 \times 10 \times 7 \mathrm{~km}^{3}$, centered on the tornado or low-level mesocyclone (prior to tornadogenesis) for all volumes available from 2046 to 2116 UTC (the end of data collection). The maximum distance between the radar and the tornado during this timeframe was $\sim 13 \mathrm{~km}$, while the minimum was $\sim 3 \mathrm{~km}$. To retain consistency between analyses, we chose the grid spacing to represent the worst-case scenario of a distance of $13 \mathrm{~km}$. The Barnes's smoothing parameter $\kappa$ was therefore chosen to be $0.688 \mathrm{~km}^{2}$ based upon Pauley and $\mathrm{Wu}(1990)$ who recommend that $\kappa$ equal 1.33 $\left(\theta_{b} \times R_{\max }\right)^{2}$, where $\theta_{b}$ is the beamwidth of the radar in radians and $R_{\max }$ is the maximum distance desired in your grid domain. According to Koch et al. (1983), the grid spacing was chosen to be approximately equal to $\left(\theta_{b} \times R_{\max }\right) / 2.5$. This procedure is consistent with a grid resolution of $100 \times 100 \times 100 \mathrm{~m}^{3}(x, y, z)$. This choice, however, implies that the grids are technically underdetermined in grid regions away from the tornado. However, since the area of interest was the tornado in these analyses, this issue is considered unimportant. An advection correction scheme was implemented on these analyses. Storm motion was calculated on the basis of the translation of the tornado signature during a 2-min period centered on the analysis time, and the data were advected linearly either forward or backward with respect to the storm motion vector, to the central grid time. This process removes any spurious tilt or shape to features caused by storm translation.

A second objective analysis was performed using a modified Barnes's scheme (modified to include an attenuation factor), with different specifications to prepare the data for a dual-Doppler analysis (DDA) synthesized at 2056 UTC to investigate the horizontal winds in the vicinity of the weak-echo reflectivity band mentioned in section 4d (Fig. 1). Data from a second rapid-scan radar [the mobile weather radar $2005 \mathrm{X}$-band phased array (MWR-05XP; Bluestein et al. (2010); French et al. 2014)] were used with the RaXPol data to generate the dual-Doppler synthesis. Two-dimensional objective and dual-Doppler analyses were synthesized at 2056:04 UTC, which was chosen based on when the last MWR-05XP scan was available, and represents the time closest to the development of the WRB. At this time, the tornado was approximately $15 \mathrm{~km}$ from the MWR, but only $\sim 4 \mathrm{~km}$ away from RaXPol. Because the distance was greater for the MWR and because it has a coarser beamwidth $\left(1.4^{\circ}\right)$, the objectively analyzed grid needed to be coarser. Here, a $200 \times 200 \times 200 \mathrm{~m}^{3}$ grid 
was used following the same procedure described above and a $\kappa$ value of $0.178 \mathrm{~km}^{2}$. Because the MWR-05XP transmits multiple beams simultaneously in the vertical while scanning horizontally, data from all elevation angles used were acquired nearly simultaneously as well; one complete volume scan took $11 \mathrm{~s}$. The RaXPol scan was completed in $2 \mathrm{~s}$. Therefore, no advection correction was applied to this analysis.

Several factors inhibited the construction of quantitative volumetric analyses during the last two minutes of MWR-05XP data collection. These factors are as follows: 1) MWR-05XP was not leveled during deployment and the pitch and roll of the instrument were not recorded; 2) MWR-05XP was located $11.8 \mathrm{~km}$ eastnortheast of RaXPol, farther away from the storm, and had coarser azimuthal resolution (the 3-dB beamwidth is $2.0^{\circ}$ ), creating a mismatch in sampling volumes between the two instruments; 3) RaXPol switched to rapid-scan single elevation mode at 2055 UTC, making volumetric analyses unavailable after this time; and 4) the height of the beams from each instrument at the tornado was offset by $\sim 150 \mathrm{~m}$ when RaXPol switched to $1^{\circ}$ single-elevation PPIs.

The contributions to error because MWR-05XP was unlevel were evaluated by constructing a series of sensitivity tests using a single RaXPol PPI acquired during RaXPol's volume scans and varying the MWR-05XP input elevation angle by $\pm 3^{\circ}$. This simulates the effects that a roll and pitch angle of $3^{\circ}$ would have on the analyses. A visit to the MWR-05XP deployment location after the event indicated that $3^{\circ}$ is likely the greatest tilt that would have existed. Analyses were performed for a height of $1 \mathrm{~km}$ ARL at the location of the tornado. This height corresponds with a RaXPol elevation angle of $12^{\circ}$ and an MWR-05XP reference elevation angle of $4^{\circ}$ (the elevation angle for which the beam would be located at $1 \mathrm{~km}$ if the radar were truly level). While there were sometimes significant differences in the magnitude and direction of the synthesized horizontal winds (8$16 \mathrm{~m} \mathrm{~s}^{-1}$ and $20^{\circ}-30^{\circ}$, respectively), the qualitative features of the analyses remained mostly unchanged. The locations of the RFGF and tornado, for example, were consistent between analyses. While it is acknowledged that there are undoubtedly errors in the DDAs as a result of the factors described above, we conclude that the analyses are still useful for qualitative purposes.

The $1^{\circ}$ data were used for both instruments (the lowest elevation angle available for the MWR-05XP). As indicated above, the beam height was off by about $150 \mathrm{~m}$ between the two instruments. However, the critical radius specified in the objective analysis schemes was $500 \mathrm{~m}$ so we do not consider this to be a detrimental source of error, considering the analysis is purely for qualitative purposes. The dual-Doppler analysis was performed using NCAR's CEDRIC software, and only horizontal wind vectors were calculated. The horizontal wind components (Armijo 1969) were estimated by iteratively solving for the projection of motion along the radial wind until the solutions converged between iterations. To minimize errors in the calculations of $U$ and $V$, velocity data lying within $\pm 20^{\circ}$ from the baseline between RaXPol and MWR-05XP were removed. Finally, the objectively analyzed reflectivity and radial velocity fields were assimilated into the dual-Doppler analyses to provide the objectively analyzed reflectivity and radial velocity data for both RaXPol and MWR-05XP.

\section{REFERENCES}

Alexander, C. R., and J. Wurman, 2005: The 30 May 1998 Spencer, South Dakota, storm. Part I: The structural evolution and environment of the tornadoes. Mon. Wea. Rev., 133, 72-97, doi:10.1175/MWR-2855.1.

Armijo, L., 1969: A theory for the determination of wind and precipitation velocities with Doppler radars. J. Atmos. Sci., 26, 570-573, doi:10.1175/1520-0469(1969)026<0570: ATFTDO $>2.0 . \mathrm{CO} ; 2$.

Barnes, S. L., 1964: A technique for maximizing details in numerical weather map analysis. J. Appl. Meteor., 3, 396-409, doi:10.1175/1520-0450(1964)003<0396:ATFMDI > 2.0.CO;2.

Bluestein, H. B., and W. P. Unruh, 1989: Observations of the wind field in tornadoes, funnel clouds, and wall clouds with a portable Doppler radar. Bull. Amer. Meteor. Soc., 70, 1514-1525, doi:10.1175/1520-0477(1989)070<1514:OOTWFI >2.0.CO;2.

— radar for tornado research. The Tornado: Its Structure, Dynamics, Prediction, and Hazards, Geophys. Monogr., Vol. 79, Amer. Geophys. Union, 367-376.

, and A. L. Pazmany, 2000: Observations of tornadoes and other convective phenomena with a mobile, 3-mm wavelength, Doppler radar: The spring 1999 field experiment. Bull. Amer. Meteor. Soc., 81, 2939-2951, doi:10.1175/1520-0477(2000)081<2939: OOTAOC $>2.3 . \mathrm{CO} ; 2$.

_ , and R. M. Wakimoto, 2003: Mobile radar observations of severe convective storms. Radar and Atmospheric Science: A Collection of Essays in Honor of David Atlas, Meteor. Monogr., No. 52, 105-105, doi:10.1175/0065-9401(2003)030<0105: MROOSC $>2.0 . \mathrm{CO} ; 2$.

— A. L. Pazmany, J. C. Galloway, and R. E. Mcintosh, 1995: Studies of the substructure of severe convective storms using a mobile 3-mm-wavelength Doppler radar. Bull. Amer. Meteor. Soc., 76, 2155-2169, doi:10.1175/1520-0477(1995)076<2155: SOTSOS $>2.0 . \mathrm{CO} ; 2$.

— C. C. Weiss, and A. L. Pazmany, 2003: Mobile Doppler radar observations of a tornado in a supercell near Bassett, Nebraska, on 5 June 1999. Part I: Tornadogenesis. Mon. Wea. Rev., 131, 2954-2967, doi:10.1175/1520-0493(2003)131<2954: MDROOA $>2.0 . \mathrm{CO} ; 2$.

,$- \ldots$, and ——, 2004: The vertical structure of a tornado near Happy, Texas, on 5 May 2002: High-resolution, mobile, W-band, Doppler radar observations. Mon. Wea. Rev., 132, 2325-2337, doi:10.1175/1520-0493(2004)132<2325: TVSOAT $>2.0 . \mathrm{CO} ; 2$. 
—, M. M. French, R. L. Tanamachi, S. Frasier, K. Hardwick, F. Junyent, and A. L. Pazmany, 2007: Close-range observations of tornadoes in supercells made with a dual-polarization, X-band, mobile Doppler radar. Mon. Wea. Rev., 135, 15221543, doi:10.1175/MWR3349.1.

,-- I. PopStefanija, R. T. Bluth, and J. B. Knorr, 2010: A mobile, phased-array Doppler radar for the study of severe convective storms: The MWR-05XP. Bull. Amer. Meteor. Soc., 91, 579-600, doi:10.1175/2009BAMS2914.1.

_ J. C. Snyder, and J. B. Houser, 2015: A multi-scale overview of the El Reno, Oklahoma, tornadic supercell of 31 May 2013. Wea. Forecasting, 30, 525-552, doi:10.1175/WAF-D-14-00152.1.

— M. M. French, J. C. Snyder, and J. B. Houser, 2016: Doppler radar observations of anticyclonic tornadoes in cyclonically rotating, right-moving supercells. Mon. Wea. Rev., 144, 15911616, doi:10.1175/MWR-D-15-0304.1.

Bodine, D. J., R. D. Palmer, and G. Zhang, 2014: Dual-wavelength polarimetric radar analyses of tornadic debris signatures. J. Appl. Meteor. Climatol., 53, 242-261, doi:10.1175/JAMC-D-13-0189.1.

Burgess, D., M. A. Magsig, J. Wurman, D. C. Dowell, and Y. Richardson, 2002: Radar observations of the 3 May 1999 Oklahoma City tornado. Wea. Forecasting, 17, 456-471, doi:10.1175/1520-0434(2002)017<0456:ROOTMO>2.0.CO;2.

Byko, Z., P. Markowski, Y. Richardson, J. Wurman, and E. Adlerman, 2009: Descending reflectivity cores in supercell thunderstorms observed by mobile radars and in a highresolution numerical simulation. Wea. Forecasting, 24, 155186, doi:10.1175/2008WAF2222116.1.

Cheong, B. L., R. Kelley, R. D. Palmer, Y. Zhang, M. Yeary, and T.-Y. Yu, 2013: PX-1000: A solid-state polarimetric X-band weather radar and time-frequency multiplexed waveform for blind range mitigation. IEEE Trans. Instrum. Meas., 62, 30643072, doi:10.1109/TIM.2013.2270046.

Church, C. R., J. T. Snow, G. L. Baker, and E. M. Agee, 1979: Characteristics of tornado-like vortices as a function of swirl ratio: A laboratory investigation. J. Atmos. Sci., 36, 1755-1776, doi:10.1175/1520-0469(1979)036<1755: COTLVA $>2.0 . \mathrm{CO} ; 2$.

Dawson, D. T., E. R. Mansell, Y. Jung, L. J. Wicker, M. R. Kumjian, and M. Xue, 2014: Low-level $Z_{\mathrm{DR}}$ signatures in supercell forward flanks: The role of size sorting and melting of hail. J. Atmos. Sci., 71, 276-299, doi:10.1175/JAS-D-13-0118.1.

Doviak, R. J., and D. S. Zrnić, 2006: Doppler Radar and Weather Observations. 2nd ed. Dover Publishing Inc., 562 pp.

Dowell, D. C., C. R. Alexander, J. M. Wurman, and L. J. Wicker, 2005: Centrifuging of hydrometeors and debris in tornadoes: Radar-reflectivity patterns and wind-measurement errors. Mon. Wea. Rev., 133, 1501-1524, doi:10.1175/MWR2934.1.

Edwards, R., J. G. Ladue, J. T. Ferree, K. Scharfenberg, C. Maier, and W. L. Coulbourne, 2013: Tornado intensity estimation past, present, and future. Bull. Amer. Meteor. Soc., 94, 641653, doi:10.1175/BAMS-D-11-00006.1.

French, M. M., H. B. Bluestein, I. Popstefanija, C. A. Baldi, and R. T. Bluth, 2013: Reexamining the vertical development of tornadic vortex signatures in supercells. Mon. Wea. Rev., 141, 4576-4601, doi:10.1175/MWR-D-12-00315.1.

$-\longrightarrow,-,-$, and,- 2014 : Mobile, phased-array, Doppler radar observations of tornadoes at X-band. Mon. Wea. Rev., 142, 1010-1036, doi:10.1175/MWR-D-13-00101.1. , P. S. Skinner, L. J. Wicker, and H. B. Bluestein, 2015: Documenting a rare tornado merger observed in the 24 May 2011 El Reno-Piedmont, Oklahoma, supercell. Mon. Wea. Rev., 143, 3025-3043, doi:10.1175/MWR-D-14-00349.1.
Fujita, T., 1981: Tornadoes and downbursts in the context of generalized planetary scales. J. Atmos. Sci., 38, 1511-1534, doi:10.1175/1520-0469(1981)038<1511:TADITC > 2.0.CO;2.

Grzych, M. L., B. D. Lee, and C. A. Finley, 2007: Thermodynamic analysis of supercell rear-flank downdrafts from project ANSWERS. Mon. Wea. Rev., 135, 240-246, doi:10.1175/ MWR3288.1.

Hirth, B. D., J. L. Schroeder, and C. C. Weiss, 2008: Surface analysis of the rear-flank downdraft outflow in two tornadic supercells. Mon. Wea. Rev., 136, 2344-2363, doi:10.1175/ 2007MWR2285.1.

Houser, J. B., H. B. Bluestein, and J. C. Snyder, 2015: Rapid-scan, polarimetric, Doppler radar observations of tornadogenesis and tornado dissipation in a tornadic supercell: The "El Reno, Oklahoma" storm of 24 May 2011. Mon. Wea. Rev., 143, 26852710, doi:10.1175/MWR-D-14-00253.1.

Illingworth, A. J., J. W. F. Goddard, and S. M. Cherry, 1987: Polarization radar studies of precipitation development in convective storms. Quart. J. Roy. Meteor. Soc., 113, 469-489, doi:10.1002/qj.49711347604.

Isom, B., and Coauthors, 2013: The Atmospheric Imaging Radar: Simultaneous volumetric observations using a phased array weather radar. J. Atmos. Oceanic Technol., 30, 655-675, doi:10.1175/JTECH-D-12-00063.1.

Jameson, A. R., M. J. Murphy, and E. P. Krider, 1996: Multiple parameter radar observations of isolated Florida thunderstorms during the onset of electrification. J. Appl. Meteor., 35, 343-354, doi:10.1175/1520-0450(1996)035<0343: MPROOI $>2.0 . \mathrm{CO} ; 2$.

Johnstone, T., S. E. Nelson, L. Belanger, M. E. Anderson, K. B. Laws, B. Carcione, C. J. Schultz, and L. D. Carey, 2012: Operational use of polarimetric tornadic debris signatures. 26th Conf. on Severe Local Storms, Nashville, TN, Amer. Meteor. Soc., 16.5. [Available online at https://ams.confex.com/ams/ 26SLS/webprogram/Paper211567.html.]

Kennedy, A., J. M. Straka, and E. N. Rasmussen, 2007: A statistical study of the association of DRCs with supercells and tornadoes. Wea. Forecasting, 22,1191-1199, doi:10.1175/2007WAF2006095.1.

Koch, S. E., M. DesJardins, and P. J. Kocin, 1983: An interactive Barnes objective map analysis scheme for use with satellite and conventional data. J. Climate Appl. Meteor., 22, 1487-1503, doi:10.1175/1520-0450(1983)022<1487:AIBOMA > 2.0.CO;2.

Kosiba, K. A., and J. Wurman, 2010: The three-dimensional axisymmetric wind field structure of the Spencer, South Dakota, 1998 tornado. J. Atmos. Sci., 67, 3074-3083, doi:10.1175/ 2010JAS3416.1.

,-- , Y. Richardson, P. Markowski, P. Robinson, and J. Marquis, 2013: Genesis of the Goshen County, Wyoming, tornado on 5 June 2009 during VORTEX2. Mon. Wea. Rev., 141, 1157-1181, doi:10.1175/MWR-D-12-00056.1.

Kumjian, M. R., and A. V. Ryzhkov, 2008: Polarimetric signatures in supercell thunderstorms. J. Appl. Meteor. Climatol., 47, 1940-1961, doi:10.1175/2007JAMC1874.1.

— A. P. Khain, N. Benmoshe, E. Ilotoviz, A. V. Ryzhkov, and V. T. J. Phillips, 2014: The anatomy and physics of $Z_{D R}$ columns: Investigating a polarimetric radar signature with a spectral bin microphysical model. J. Appl. Meteor. Climatol., 53, 1820-1843, doi:10.1175/JAMC-D-13-0354.1.

Kurdzo, J. M., D. J. Bodine, B. L. Cheong, and R. D. Palmer, 2015: High-temporal resolution polarimetric X-band Doppler radar observations of the 20 May 2013 Moore, Oklahoma, tornado. Mon. Wea. Rev., 143, 2711-2735, doi:10.1175/ MWR-D-14-00357.1. 
Lee, B. D., C. A. Finley, and C. D. Karstens, 2012: The Bowdle, South Dakota, cyclic tornadic supercell of 22 May 2010: Surface analysis of rear-flank downdraft evolution and multiple internal surges. Mon. Wea. Rev., 140, 3419-3441, doi:10.1175/ MWR-D-11-00351.1.

Lewellen, D. C., W. S. Lewellen, and J. Xia, 2000: The influence of a local swirl ratio on tornado intensification near the surface. J. Atmos. Sci., 57, 527-544, doi:10.1175/ 1520-0469(2000)057<0527:TIOALS >2.0.CO;2.

Marquis, J., Y. Richardson, J. Wurman, and P. Markowski, 2008: Single- and dual-Doppler analysis of a tornadic vortex and surrounding storm-scale flow in the Crowell, Texas, supercell of 30 April 2000. Mon. Wea. Rev., 136, 5017-5043, doi:10.1175/ 2008MWR2442.1.

$\longrightarrow,-,-,-$, D. Dowell, and J. Wurman, 2012: Tornado maintenance investigated with high-resolution dual-Doppler and EnKF analysis. Mon. Wea. Rev., 140, 3-27, doi:10.1175/ MWR-D-11-00025.1.

McDonald, J. R., and K. C. Mehta, 2006: A recommendation for an Enhanced Fujita Scale (EF-Scale). National Weather Service Rep., Wind Science and Engineering Center, Texas Tech University, Lubbock, TX, 111 pp. [Available online at http:// www.depts.ttu.edu/nwi/Pubs/FScale/EFScale.pdf.]

Palmer, R. D., and Coauthors, 2011: Observations of the 10 May 2010 tornado outbreak using OU-PRIME: Potential for new science with high-resolution polarimetric radar. Bull. Amer. Meteor. Soc., 92, 871-891, doi:10.1175/2011BAMS3125.1.

Pauley, P. M., and X. Wu, 1990: The theoretical, discrete, and actual response of the Barnes objective analysis scheme for oneand two-dimensional fields. Mon. Wea. Rev., 118, 1145-1163, doi:10.1175/1520-0493(1990)118<1145:TTDAAR > 2.0.CO;2.

Pauley, R. L., and J. T. Snow, 1988: On the kinematics and dynamics of the 18 July 1986 Minneapolis tornado. Mon. Wea. Rev., 116, 2731-2736, doi:10.1175/1520-0493(1988)116<2731: OTKADO $>2.0 . \mathrm{CO} ; 2$.

Pazmany, A. L., J. B. Mead, H. B. Bluestein, J. C. Snyder, and J. B. Houser, 2013: A mobile rapid-scanning X-band polarimetric (RaXPol) Doppler radar system. J. Atmos. Oceanic Technol., 30, 1398-1413, doi:10.1175/JTECH-D-12-00166.1.

Rasmussen, E. N., J. M. Straka, M. G. Gilmore, and R. DaviesJones, 2006: A preliminary survey of rear-flank descending reflectivity cores in supercell storms. Wea. Forecasting, 21, 923-938, doi:10.1175/WAF962.1.

Romine, G. S., D. W. Burgess, and R. B. Wilhelmson, 2008: A dualpolarization-radar-based assessment of the 8 May 2003 Oklahoma City area tornadic supercell. Mon. Wea. Rev., 136, 2849-2870, doi:10.1175/2008MWR2330.1.

Ryzhkov, A. V., T. J. Schuur, D. W. Burgess, and D. S. Zrnić, 2005: Polarimetric tornado detection. J. Appl. Meteor., 44, 557-570, doi:10.1175/JAM2235.1.

Saari, M. D. W., R. M. Lawton, C. J. Schultz, and L. D. Carey, 2014: Early characteristics of the polarimetric tornadic debris signature associated with the 20 May 2013 Newcastle-Moore, Oklahoma, tornado. Electron. J. Oper. Meteor., 2, 110-114, doi:10.15191/nwajom.2014.0210.

Schenkman, A. D., M. Xue, and M. Hu, 2014: Tornadogenesis in a high-resolution simulation of the 8 May 2003 Oklahoma City supercell. J. Atmos. Sci., 71, 130-154, doi:10.1175/ JAS-D-13-073.1.

Schultz, C. J., and Coauthors, 2012: Dual-polarization tornadic debris signatures. Part II: Comparisons and caveats. Electron. J. Oper. Meteor., 13, 128-150. [Available online at www.nwas. org/ej/pdf/2012-EJ10.pdf.]
Shapiro, A., 2001: A centrifugal wave solution of the Euler and Navier-Stokes equations. Z. Angew. Math. Phys., 52, 913-923, doi:10.1007/PL00001587.

Skinner, P. S., C. C. Weiss, M. M. French, H. B. Bluestein, P. M. Markowski, and Y. P. Richardson, 2014: VORTEX2 observations of a low-level mesocyclone with multiple internal rearflank downdraft momentum surges in the 18 May 2010 Dumas, Texas, supercell. Mon. Wea. Rev., 142, 2935-2960, doi:10.1175/ MWR-D-13-00240.1.

Snyder, J. C., and H. B. Bluestein, 2014: Some considerations for the use of mobile Doppler radar data for tornado intensity determination. Wea. Forecasting, 29, 799-827, doi:10.1175/ WAF-D-14-00026.1.

,,-- G. Zhang, and S. J. Frasier, 2010: Attenuation correction and hydrometeor classification of high-resolution, $\mathrm{X}$-band, dual-polarized mobile radar measurements in severe convective storms. J. Atmos. Oceanic Technol., 27, 1979-2001, doi:10.1175/2010JTECHA1356.1.

, — _ V. Venkatesh, and S. J. Frasier, 2013: Observations of polarimetric signatures in supercells by an X-band mobile Doppler radar. Mon. Wea. Rev., 141, 3-29, doi:10.1175/MWR-D-12-00068.1. —, A. V. Ryzhkov, M. R. Kumjian, A. P. Khain, and J. C. Picca, 2015: A $Z_{D R}$ column detection algorithm to examine convective storm updrafts. Wea. Forecasting, 30, 1819-1844, doi:10.1175/WAF-D-15-0068.1.

Tanamachi, R. L., and P. L. Heinselman, 2016: Rapid-scan, polarimetric observations of central Oklahoma severe storms on 31 May 2013. Wea. Forecasting, 31, 19-42, doi:10.1175/ WAF-D-15-0111.1.

, H. B. Bluestein, J. B. Houser, S. J. Frasier, and K. M. Hardwick, 2012: Mobile, X-band, polarimetric Doppler radar observations of the 4 May 2007 Greensburg, Kansas, tornadic supercell. Mon. Wea. Rev., 140, 2103-2125, doi:10.1175/ MWR-D-11-00142.1.

Van Den Broeke, M. S., 2015: Polarimetric tornadic debris signature variability and debris fallout signatures. J. Appl. Meteor. Climaol., 54, 2389-2405, doi:10.1175/JAMC-D-15-0077.1.

— , and S. T. Jauernic, 2014: Spatial and temporal characteristics of polarimetric tornadic debris signatures. J. Appl. Meteor. Climatol., 53, 2217-2231, doi:10.1175/JAMC-D-14-0094.1.

Wakimoto, R., N. T. Atkins, and J. Wurman, 2011: The LaGrange tornado during VORTEX2. Part I: Photogrammetric analysis of the tornado combined with single-Doppler radar data. Mon. Wea. Rev., 139, 2233-2258, doi:10.1175/ 2010MWR3568.1.

— P. Stauffer, W. C. Lee, N. T. Atkins, and J. Wurman, 2012: Finescale structure of the LaGrange, Wyoming, tornado during VORTEX2: GBVTD and photogrammetric analyses. Mon. Wea. Rev., 140, 3397-3418, doi:10.1175/MWR-D-12-00036.1.

K. M. Butler, H. B. Bluestein, K. Thiem, J. Snyder, and J. Houser, 2015: Photogrammetric analysis of the 2013 El Reno tornado combined with mobile X-Band polarimetric radar data. Mon. Wea. Rev., 143, 2657-2683, doi:10.1175/ MWR-D-15-0034.1.

Wakimoto, R. M., and Coauthors, 2016: Aerial damage survey of the 2013 El Reno tornado combined with mobile radar data. Mon. Wea. Rev., 144, 1749-1776, doi:10.1175/MWR-D-15-0367.1.

Wurman, J., 2002: The multiple-vortex structure of a tornado. Wea. Forecasting, 17, 473-505, doi:10.1175/1520-0434(2002)017<0473: TMVSOA $>2.0 . \mathrm{CO} ; 2$.

, and M. Randall, 2001: An inexpensive, mobile, rapid-scan radar. Preprints, 30th Conf. on Radar Meteorology, Munich, Germany, Amer. Meteor. Soc., 98-100. 
, and C. R. Alexander, 2005: The 30 May 1998 Spencer, South Dakota, storm. Part II: Comparison of observed damage and radar-derived winds in the tornadoes. Mon. Wea. Rev., 133, 97-119, doi:10.1175/MWR-2856.1.

— and mesocyclone structures. Wea. Forecasting, 28, 1157-1174, doi:10.1175/WAF-D-12-00127.1.

_ J. Straka, E. Rasmussen, M. Randall, and A. Zahrai, 1997: Design and deployment of a portable, pencil-beam, pulsed, 3-cm Doppler radar. J. Atmos. Oceanic Technol., 14, 1502-1512, doi:10.1175/1520-0426(1997)014<1502:DADOAP>2.0.CO;2.

, Y. Richardson, C. Alexander, S. Weygandt, and P. F. Zhang, 2007: Dual-Doppler and single-Doppler analysis of a tornadic storm undergoing mergers and repeated tornadogenesis. Mon. Wea. Rev., 135, 736-758, doi:10.1175/ MWR3276.1.

_ video observations of the interior structure of a tornado and the wind-damage relationship. Bull. Amer. Meteor. Soc., 94, 835-846, doi:10.1175/BAMS-D-12-00114.1.

,,,--- and T. Marshall, 2014: The role of multiple-vortex tornado structure in causing storm researcher fatalities. Bull. Amer. Meteor. Soc., 95, 31-45, doi:10.1175/BAMS-D-13-00221.1.

Zrnić, D. S., and Coauthors, 2007: Agile-beam phased array radar for weather observations. Bull. Amer. Meteor. Soc., 88, 1753 1766, doi:10.1175/BAMS-88-11-1753. 